JOURNAL OF THE

AMERICAN MATHEMATICAL SOCIETY

Volume 26, Number 4, October 2013, Pages 1025-1050

S 0894-0347(2013)00768-7

Article electronically published on March 27, 2013

\title{
LOCALIZING VIRTUAL CYCLES BY COSECTIONS
}

\author{
YOUNG-HOON KIEM AND JUN LI
}

\section{INTRODUCTION}

Invariants defined by virtual cycles of moduli spaces have played important roles in research in algebraic geometry. Invariants of this kind include the much studied Gromov-Witten (GW for short) invariants of varieties and the recently introduced Donaldson-Thomas (DT for short) invariants of Calabi-Yau three-folds.

One of the main challenges in studying such invariants is to develop techniques for investigating virtual cycles. In this paper, we will present a new technique, which we call localization by a cosection of the obstruction sheaf (Theorem 1.1). This is achieved after constructing a localized Gysin map (Proposition 1.3).

Theorem 1.1 (Localization by a cosection). Let $\mathcal{M}$ be a Deligne-Mumford stack endowed with a perfect obstruction theory. Suppose the obstruction sheaf $\mathcal{O b}_{\mathcal{M}}$ admits a surjective homomorphism $\sigma:\left.\mathcal{O}_{\mathcal{M}}\right|_{U} \rightarrow \mathcal{O}_{U}$ over an open $U \subset \mathcal{M}$. Let $\mathcal{M}(\sigma)=\mathcal{M} \backslash U$. Then $(\mathcal{M}, \sigma)$ has a localized virtual cycle

$$
[\mathcal{M}]_{\text {loc }}^{\mathrm{vir}} \in A_{*} \mathcal{M}(\sigma) .
$$

This cycle satisfies the usual properties of virtual cycles; it relates to the usual virtual cycle $[\mathcal{M}]^{\text {vir }}$ via $[\mathcal{M}]^{\text {vir }}=\iota_{*}[\mathcal{M}]_{\text {loc }}^{\text {vir }} \in A_{*} \mathcal{M}$, where $\iota: \mathcal{M}(\sigma) \rightarrow \mathcal{M}$ is the inclusion.

This work originated from our effort to understand Lee-Parker's discovery that GW-invariants of surfaces with holomorphic two-forms localize along the loci of stable maps to canonical divisors [21. We show that a holomorphic two-form induces a cosection of the obstruction sheaf of the moduli space; applying localization by cosection, we recover and generalize Lee-Parker's theorem as follows.

Let $X$ be a smooth quasi-projective variety with a holomorphic two-form $\theta \in$ $H^{0}\left(\Omega_{X}^{2}\right)$. Let $\overline{\mathcal{M}}_{g, n}(X, \beta)$ denote the moduli stack of $n$-pointed stable maps of genus $g$ to $X$ with homology class $\beta$. The two-form $\theta$ on $X$ induces a cosection $\sigma$ of the obstruction sheaf of $\overline{\mathcal{M}}_{g, n}(X, \beta)$; the degeneracy loci $\mathcal{M}(\sigma)$ consist of stable maps $[u: C \rightarrow X]$ satisfying $\theta\left(u_{*} T C\right)=0$ (called $\theta$-null stable maps) where $\theta$ is viewed as an antisymmetric homomorphism $T_{X} \rightarrow \Omega_{X}$. Applying the localization by cosection, we obtain

Theorem 1.2. For a pair $(X, \theta)$ of a smooth quasi-projective variety and a holomorphic two-form, the virtual fundamental class of $\overline{\mathcal{M}}_{g, n}(X, \beta)$ vanishes unless $\beta$ is represented by a $\theta$-null stable map.

Received by the editors February 11, 2011 and, in revised form, November 28, 2012.

2010 Mathematics Subject Classification. Primary 14N35.

The first author was partially supported by NRF grant 2011-0027969.

The second author was partially supported by NSF grant NSF-0601002. 
The localization by cosection has other applications in the study of GW-invariants and of DT-invariants. One is the work on quantum cohomology of the Hilbert scheme of points by W.-P. Li and the second author of this paper. Using meromorphic two-forms of algebraic surfaces, they determined the two-point extreme quantum cohomology of the Hilbert scheme of points of any algebraic surface [23.

A special but important case is when the cosection is regular and surjective everywhere. In this case, a reduced virtual cycle can be defined using localization by a cosection. The notion of reduced virtual fundamental class was first introduced by Okounkov-Pandharipande in [29, §3.4.3] for GW-invariants of holomorphic symplectic varieties. However, the idea was already around before, e.g., in the work of Donaldson [8], Lee [19, Bryan-Leung [3, 4], and J. Li [22]. In studying curve counting [28, Maulik-Pandharipande-Thomas use localization of the virtual cycle by a cosection to define the reduced Gromov-Witten and Pandharipande-Thomas invariants of a class of Calabi-Yau three-folds; in the appendix of the same paper, they provide an in-depth discussion on the relationships between the reduced class construction and usual deformation-obstruction theories.

Another application of reduced virtual cycles appears in a recent paper [15]: the authors use localization by a cosection to prove a $\mathbb{C}^{*}$-wall crossing formula of DT-invariants by producing a reduced virtual fundamental class of the master space.

Quite recently, H.-L. Chang and the second author of this paper, in [6], used the cosection localization to define the Gromov-Witten invariants of stable maps to $\mathbb{P}^{4}$ with $p$-fields and related them to those of stable maps to a quintic three-fold.

The proof of localization by a cosection (Theorem 1.1) consists of two parts. In one part, we prove that the intrinsic normal cone of the perfect obstruction theory of $\mathcal{M}$ lies in the kernel cone stack of the cosection $\sigma$. In particular, if there is a surjective homomorphism $\sigma: \mathcal{O} b_{\mathcal{M}} \rightarrow \mathcal{L}$ to a locally free sheaf $\mathcal{L}$, the intrinsic normal cone lies in a smaller cone stack and we can define a reduced virtual fundamental class.

The other part of the proof is the construction of the following localized Gysin map.

Proposition 1.3 (Localized Gysin Map). Let $E$ be a rank $r$ vector bundle on a Deligne-Mumford stack $\mathcal{M}$, and let $\sigma$ be a meromorphic surjective cosection of $E$, meaning that there is an open $U \subset \mathcal{M}$ so that $\sigma$ is a surjective homomorphism $\sigma:\left.E\right|_{U} \rightarrow \mathcal{O}_{U}$. Let $\mathcal{M}(\sigma)=\mathcal{M} \backslash U$ and let $E(\sigma)=\left.E\right|_{\mathcal{M}(\sigma)} \cup \operatorname{ker}\left\{\sigma:\left.E\right|_{U} \rightarrow \mathcal{O}_{U}\right\}$. The basic construction stated in Section 2 defines a homomorphism

$$
s_{E, \sigma}^{!}: A_{*} E(\sigma) \rightarrow A_{*-r} \mathcal{M}(\sigma),
$$

which we call the localized Gysin map. Furthermore, if we denote the inclusions by $\iota: \mathcal{M}(\sigma) \rightarrow \mathcal{M}, \tilde{\iota}: E(\sigma) \rightarrow E$, and let $s_{E}: A_{*} E \rightarrow A_{*-r} \mathcal{M}$ be the usual Gysin map of intersecting with the zero section of $E$, then we have

$$
\iota_{*} \circ s_{E, \sigma}^{!}=s_{E}^{!} \circ \tilde{\iota}_{*}: A_{*} E(\sigma) \rightarrow A_{*-r} \mathcal{M} .
$$

By applying the localized Gysin map to the intrinsic normal cone in $E(\sigma)$, we obtain the localized virtual cycle $[\mathcal{M}]_{\text {loc }}^{\text {vir }}$ in Theorem 1.1 .

The paper is organized as follows. In Sections 2 and 3, we construct the localized Gysin map. In Section 4, we show that the intrinsic normal cone lies in the kernel cone stack. In Section 5, we define the localized virtual cycle and prove its deformation invariance. An application of this localization technique to $\mathrm{GW}$-invariants 
of varieties with holomorphic two-forms is presented in Section 6. In Section 7, we discuss other possible applications. In the Appendix, we give an analytic definition of the localized Gysin map and prove its equivalence with the algebraic definition.

Addendum. The current version is the replacement of the first half of [12]. Our prior treatment of localization by a cosection used a topological definition of the localized Gysin map [12, which limits its application in algebraic geometry. In this paper, we provide an algebraic construction of the localized Gysin map, including the Chow groups of the total space of a cone-stack over a Deligne-Mumford stack. This makes it possible to directly apply other developed techniques on virtual cycles to localized virtual cycles.

In the sequel to this paper, we will prove a degeneration formula of localized GW-invariants and include its application worked out in [12, in proving MaulikPandharipande's conjecture ([27]) on degree two GW-invariants of surfaces.

Notation. In this paper, all schemes and stacks are defined over the complex number field $\mathbb{C}$. We will use $Z_{*} X$ (resp. $A_{*} X$; resp. $W_{*} X$ ) to denote the group of algebraic cycles (resp. group of algebraic cycles modulo rational equivalence; resp. group of rational equivalences) with $\mathbb{Q}$-coefficients.

Since we will be working with locally free sheaves and cycles in the total spaces of the vector bundles associated to the locally free sheaves, to streamline the notation, we will use the same symbol to denote a locally free sheaf as well as its associated vector bundle. Thus, given a vector bundle (locally free sheaf) $E$, by $Z_{*} E$ we mean the group of cycles of the total space of $E$, and by $E \rightarrow \mathcal{O} b_{\mathcal{M}}$ we mean a sheaf homomorphism $\mathcal{O}_{\mathcal{M}}(E) \rightarrow \mathcal{O} b_{\mathcal{M}}$.

Given a subvariety $T \subset E$, we denote by $[T] \in Z_{*} E$ its associated cycle and denote by $[T] \in A_{*} E$ its rational equivalence class in $A_{*} E$.

\section{LOCALIZED Gysin MAPS}

Let $\pi: E \rightarrow \mathcal{M}$ be a rank $r$ vector bundle over a Deligne-Mumford (DM for short) stack $\mathcal{M}$. The usual Gysin map $s_{E}^{!}: A_{d} E \rightarrow A_{d-r} \mathcal{M}$ is defined by "intersecting" cycles in $E$ with the zero section $s_{E}$ of $E$.

In this section, we suppose that $E$ has a surjective meromorphic cosection $\sigma$.

Definition 2.1. A surjective meromorphic cosection is a surjective homomorphism $\sigma:\left.E\right|_{U} \rightarrow \mathcal{O}_{U}$ for an open substack $U \subset \mathcal{M}$.

Remark 2.2. For the purpose of defining the localized Gysin map, the degeneracy locus of a meromorphic cosection $\sigma: E-\rightarrow \mathcal{O}_{\mathcal{M}}$ includes the loci where $\sigma$ is undefined and where $\sigma$ is not surjective. In the definition above, the open $U$ is the locus where $\sigma$ is defined and surjective.

For such a $\sigma$, we let $\mathcal{M}(\sigma)=\mathcal{M} \backslash U, G=\operatorname{ker}\left\{\sigma:\left.E\right|_{U} \rightarrow \mathcal{O}_{U}\right\}$, and $E(\sigma)=$ $\left.E\right|_{\mathcal{M}(\sigma)} \cup G$, which is closed in $E$. The goal of this section is to define a localized Gysin map

$$
s_{E, \sigma}^{!}: A_{d} E(\sigma) \rightarrow A_{d-r} \mathcal{M}(\sigma)
$$

that has the usual properties of the Gysin map and coincides with $s_{E}$ when composed with the tautological $A_{d-r} \mathcal{M}(\sigma) \rightarrow A_{d-r} \mathcal{M}$. 
Definition 2.3. Let $\rho: X \rightarrow \mathcal{M}$ be a morphism from a variety $X$ to $\mathcal{M}$ such that $\rho(X) \cap U \neq \emptyset$. We call $\rho$ a $\sigma$-regularizing morphism if $\rho$ is proper, and $\rho^{*}(\sigma)$ extends to a surjective homomorphism

$$
\tilde{\sigma}: \tilde{E}:=\rho^{*} E \rightarrow \mathcal{O}_{X}(D)
$$

for a Cartier divisor $D \subset X$. We adapt the convention that $\tilde{\rho}: \tilde{E} \rightarrow E$ is the projection; $\tilde{G}:=\operatorname{ker}\{\tilde{\sigma}\} \subset \tilde{E} ;|D| \subset X$ is the support of $D$; and $\rho(\sigma):|D| \rightarrow \mathcal{M}(\sigma)$ is the $\rho$ restricted to $|D|$.

Basic construction. Let $[B] \in Z_{d} E(\sigma)$ be a cycle represented by a closed integral substack $B \subset E(\sigma)$. In case $\left.B \subset E\right|_{\mathcal{M}(\sigma)}$, we define $s_{E, \sigma}^{!_{(}}([B])=s_{\left.E\right|_{\mathcal{M}(\sigma)}}([B]) \in$ $A_{d-r} \mathcal{M}(\sigma)$. Otherwise, we pick a variety $X$ and a $\sigma$-regularizing $\rho: X \rightarrow \mathcal{M}$ such that there is a closed integral $\tilde{B} \subset \tilde{G}$ so that $\tilde{\rho}_{*}([\tilde{B}])=k \cdot[B] \in Z_{d} E$ for some $k \in \mathbb{Z}$. We define

$$
s_{E, \sigma}^{!}([B])_{\rho, \tilde{B}}=k^{-1} \cdot \rho(\sigma)_{*}\left([D] \cdot s_{\tilde{G}}^{!}([\tilde{B}])\right) \in A_{d-r} \mathcal{M}(\sigma) .
$$

Here $[D] \cdot: A_{*} X \rightarrow A_{*-1}|D|$ is the intersection with the divisor $D$.

Here is a simple example.

Example 2.4. Let $M$ be an $n$-dimensional smooth scheme, $E$ a vector bundle of rank $n$ on $M$, and $\sigma: E \rightarrow \mathcal{O}_{M}$ a cosection so that $\sigma^{-1}(0)=p$ is a simple point in $M$. Let $[M]$ be the cycle of the zero section of $E$ in $Z_{n} E(\sigma)$. Then $s_{E, \sigma}^{!}([M])=(-1)^{n}[p]$.

The proof is straightforward. Let $\rho: \tilde{M} \rightarrow M$ be the blow-up at $p$ and let $D \subset \tilde{M}$ be the exceptional divisor. We let $F=\operatorname{ker}\left\{\rho^{*} E \rightarrow \mathcal{O}_{\tilde{M}}(-D)\right\}$ and compute $s_{F}^{!}([\tilde{M}])=c_{n-1}(F)[\tilde{M}]=[D]^{n-1}$. Since $s_{E, \sigma}^{!}([M])=\rho_{*}\left([-D] \cdot s_{F}^{!}([\tilde{M}])\right)$,

$$
s_{E, \sigma}([M])=\rho_{*}\left([-D] \cdot c_{n-1}(F)\right)=\rho_{*} c_{n}\left(\rho^{*} E\right)=c_{n}(E)=(-1)^{n}[p] .
$$

See 5] for a direct application of Example 2.4

Lemma 2.5. Let the notation be as in the basic construction. Then for each closed integral $B \subset E(\sigma)$ not contained in $\left.E\right|_{\mathcal{M}(\sigma)}$, we can find a pair $(\rho, \tilde{B})$ so that $s_{E, \sigma}^{!}([B])_{\rho, \tilde{B}}$ is defined. Furthermore the resulting cycle class $s_{E, \sigma}^{!}([B])_{\rho, \tilde{B}} \in$ $A_{d-r} \mathcal{M}(\sigma)$ is independent of the choice of $(\rho, \tilde{B})$.

Proof. We let $B \subset E$ be as in the statement of the lemma; we let $B_{0}=\pi(B) \subset \mathcal{M}$, where $\pi: E \rightarrow \mathcal{M}$ is the projection. To pick $\rho$, we then pick a normal variety $X$ and a proper and generically finite morphism $\rho: X \rightarrow B_{0}$. Since $\mathcal{M}$ is a DM-stack, such a $\rho$ exists. Using $\rho$, we pull back $\tilde{E}=\rho^{*} E$, and $\rho^{*} \sigma:\left.\rho^{*} E\right|_{\rho^{-1} U} \rightarrow \mathcal{O}_{\rho^{-1} U}$. Since $B \times{ }_{\mathcal{M}} U \neq \emptyset, \rho^{-1} U \neq \emptyset$.

Next, possibly after replacing $X$ by a blow-up of $X$, we can assume that $\rho^{*} \sigma$ extends to a surjective homomorphism $\tilde{\sigma}: \tilde{E} \rightarrow \mathcal{O}_{X}(D)$ for a Cartier divisor $D \subset X$. Thus $\rho$ is a $\sigma$-regularizing morphism. We let $\tilde{\rho}: \tilde{E} \rightarrow E$ and $\tilde{G} \subset \tilde{E}$ be as defined in Definition 2.3

Since $\rho: X \rightarrow B_{0}$ is generically finite, there is an open $O \subset B_{0}$ so that $\left.\rho\right|_{\rho^{-1} O}: \rho^{-1} O \rightarrow O$ is flat and finite. We let $\tilde{B}$ be an irreducible component of $\overline{\tilde{\rho}^{-1}\left(B \cap \pi^{-1}(O)\right)}$. Since $B$ is integral and $\tilde{\rho}$ is proper, $\tilde{\rho}(\tilde{B})=B$; thus $\tilde{\rho}_{*}([\tilde{B}])=$ $k[B]$ for an integer $k$. This shows that $s_{E, \sigma}([B])_{\rho, \tilde{B}}$ is defined according to the basic construction. 
We next check that $s_{E, \sigma}^{!}([B])_{\rho, \tilde{B}}$ is independent of the choice of $(\rho, \tilde{B})$. Let $\rho^{\prime}$ : $X^{\prime} \rightarrow \mathcal{M}, \tilde{E}^{\prime}:=\rho^{*} E$, and $\tilde{B}^{\prime} \subset \tilde{E}^{\prime}$ be another choice that fulfills the requirement of the basic construction, thus giving rise to the class $s_{E, \sigma}^{!}([B])_{\rho^{\prime}, \tilde{B}^{\prime}} \in A_{d-r} \mathcal{M}(\sigma)$. We show that $s_{E, \sigma}^{!}([B])_{\rho, \tilde{B}}=s_{E, \sigma}^{!}([B])_{\rho^{\prime}, \tilde{B}^{\prime}}$.

We form $Y=X \times{ }_{\mathcal{M}} X^{\prime}$, and we denote by $q: Y \rightarrow X$ and $q^{\prime}: Y \rightarrow X^{\prime}$ the projections and by $p: Y \rightarrow \mathcal{M}$ the composite $\rho \circ q=\rho^{\prime} \circ q^{\prime}$. Since $\rho: X \rightarrow \mathcal{M}$ is generically finite and $\tilde{B} \rightarrow X$ is dominant, $\tilde{B} \times_{E} \tilde{B}^{\prime}$ contains a pure dimension $d$ irreducible component $\bar{B}$ that surjects onto $\tilde{B}$ and $\tilde{B}^{\prime}$ via the tautological projections $\tilde{q}: \bar{E}:=p^{*} E \rightarrow \tilde{E}$ and $\tilde{q}^{\prime}: \bar{E} \rightarrow \tilde{E}^{\prime}$, respectively.

We claim that there is an isomorphism $\mathcal{O}_{Y}\left(q^{*} D\right) \cong \mathcal{O}_{Y}\left(q^{\prime *} D^{\prime}\right)$ so that

$$
q^{*} \tilde{\sigma}=q^{* *} \tilde{\sigma}^{\prime}: \bar{E}=q^{*} \tilde{E}=q^{* *} \tilde{E}^{\prime} \longrightarrow \mathcal{O}_{Y}\left(q^{*} D\right) \cong \mathcal{O}_{Y}\left(q^{* *} D^{\prime}\right) .
$$

Indeed, since $\left.q^{*} \tilde{\sigma}\right|_{p^{-1} U}=\left.q^{* *} \tilde{\sigma}^{\prime}\right|_{p^{-1} U}=\left.p^{*} \sigma\right|_{p^{-1} U}$, the kernel sheaves $\left.\operatorname{ker}\left\{q^{*} \tilde{\sigma}\right\}\right|_{p^{-1} U}$ $=\left.\operatorname{ker}\left\{q^{* *} \tilde{\sigma}^{\prime}\right\}\right|_{p^{-1} U}$. Since both are subsheaves of the locally free sheaf $\bar{E}$, the two kernels are identical. Therefore, since both $q^{*} \tilde{\sigma}$ and $q^{\prime *} \tilde{\sigma}^{\prime}$ are surjective, we obtain (2.2). Furthermore, since when restricted to $p^{-1} U$ the last isomorphism in (2.2) is the identity, the arrow in (2.2) sends $1 \in \Gamma\left(\left.\mathcal{O}_{Y}\left(q^{*} D\right)\right|_{p^{-1} U}\right)$ to $1 \in \Gamma\left(\left.\mathcal{O}_{Y}\left(q^{\prime *} D^{\prime}\right)\right|_{p^{-1} U}\right)$. We conclude that $q^{*} D=q^{* *} D^{\prime}$ as divisors. We denote this divisor by $\bar{D}$.

We let $\bar{\sigma}=q^{*} \tilde{\sigma}=q^{*} \tilde{\sigma}^{\prime}$ and $\bar{G}=\operatorname{ker}\{\bar{\sigma}\}$. By (2.2), $\bar{G}$ is the pull-back of $\tilde{G}$ via $q^{*}$ and of $\tilde{G}^{\prime}=\operatorname{ker}\left\{\tilde{\sigma}^{\prime}\right\}$ via $q^{* *}$. Back to $\bar{B}$, since $\left.B \not \subset E\right|_{\mathcal{M}(\sigma)}, B \subset E(\sigma)$, and $\tilde{p}(\bar{B})=B$, we have $\bar{B} \subset \bar{G}$. We let $k_{i}$ be the integers so that

$$
\tilde{q}_{*}([\bar{B}])=k_{1}[\tilde{B}], \quad \tilde{q}_{*}^{\prime}([\bar{B}])=k_{2}\left[\tilde{B}^{\prime}\right], \quad \tilde{\rho}_{*}([\tilde{B}])=k_{3}[B], \quad \tilde{\rho}_{*}^{\prime}\left(\left[\tilde{B}^{\prime}\right]\right)=k_{4}[B] .
$$

Then since $\tilde{\rho} \circ \tilde{q}=\tilde{\rho}^{\prime} \circ \tilde{q}^{\prime}$, we have $k_{1} k_{3}=k_{2} k_{4}$.

We let $p(\sigma):|\bar{D}| \rightarrow \mathcal{M}(\sigma), q(\sigma):|\bar{D}| \rightarrow|D|$, and $\rho(\sigma):|D| \rightarrow \mathcal{M}(\sigma)$ be the restrictions of $p, q$, and $\rho$ to $|\bar{D}|$, and $|D|$, respectively. Since the projections $\tilde{q}$ and $\tilde{\rho}$ are proper and because $\tilde{q}^{-1} \tilde{G}=\bar{G}$, applying the projection formula (and using $\left.\bar{D}=q^{*} D\right)$, we obtain

$$
\begin{aligned}
s_{E, \sigma}^{!}([B])_{p, \bar{B}} & =\left(k_{1} k_{3}\right)^{-1} \cdot p(\sigma)_{*}\left([\bar{D}] \cdot s_{\bar{G}}^{!}([\bar{B}])\right)=\left(k_{1} k_{3}\right)^{-1} \cdot \rho(\sigma)_{*}\left([D] \cdot s_{\tilde{G}}^{!}\left(\left[\tilde{q}_{*} \bar{B}\right]\right)\right) \\
& =k_{3}^{-1} \cdot \rho(\sigma)_{*}\left([D] \cdot s_{\tilde{G}}^{!}([\tilde{B}])\right)=s_{E, \sigma}^{!}([B])_{\rho, \tilde{B}} .
\end{aligned}
$$

Similarly, we have $s_{E, \sigma}^{!}([B])_{p, \bar{B}}=s_{E, \sigma}^{!}([B])_{\rho^{\prime}, \tilde{B}^{\prime}}$. This proves the lemma.

The lemma shows that the basic construction defines a homomorphism

$$
s_{E, \sigma}^{!}: Z_{d} E(\sigma) \longrightarrow A_{d-r} \mathcal{M}(\sigma) .
$$

We next check that this homomorphism descends to a homomorphism $A_{d} E(\sigma) \rightarrow$ $A_{d-r} \mathcal{M}(\sigma)$. We need a simple lemma. Let $0 \rightarrow F_{1} \rightarrow F_{2} \rightarrow F_{3} \rightarrow 0$ be an exact sequence of vector bundles (locally free sheaves) on a variety $Y$. Let $r_{i}=\operatorname{rank} F_{i}$ and $p_{i}: F_{i} \rightarrow Y$ be the projections. Let $\iota: F_{1} \rightarrow F_{2}$ be the inclusion.

Lemma 2.6. For any cycle $\alpha \in A_{d} F_{1}$, we have

$$
s_{F_{2}}^{!}\left(\iota_{*} \alpha\right)=c_{r_{3}}\left(F_{3}\right) \cap s_{F_{1}}^{!}(\alpha)=s_{F_{1}}^{!}\left(c_{r_{3}}\left(F_{3}\right) \cap \alpha\right) .
$$

Proof. By [10, Thm. 3.3], there is a cycle $B \in Z_{d-r_{1}} Y$ so that $\alpha=p_{1}^{*} B \in A_{*} F_{1}$. Since $p_{1}^{*} B$ is the total space of $F_{1}$ restricted to $B$, its Segre class is $s\left(p_{1}^{*} B\right)=$ $c\left(F_{1}\right)^{-1} \cap B$, where $c\left(F_{1}\right)$ is the total Chern class of $F_{1}$. By the formula expressing the Gysin map in terms of the Segre class [10, Prop. 6.1],

$s_{F_{2}}^{!}\left(\iota_{*} \alpha\right)=s_{F_{2}}^{!}\left(p_{1}^{*} B\right)=\left[c\left(F_{2}\right) \cap\left(c\left(F_{1}\right)^{-1} \cap B\right)\right]_{d-r_{2}}=\left[c\left(F_{3}\right) \cap B\right]_{d-r_{2}}=c_{r_{3}}\left(F_{3}\right) \cap B$. 
On the other hand,

$$
s_{F_{1}}^{!}\left(c_{r_{3}}\left(F_{3}\right) \cap \alpha\right)=s_{F_{1}}^{!}\left(c_{r_{3}}\left(F_{3}\right) \cap\left[p_{1}^{*} B\right]\right)=s_{F_{1}}\left(p_{1}^{*}\left(c_{r_{3}}\left(F_{3}\right) \cap[B]\right)\right)=c_{r_{3}}\left(F_{3}\right) \cap[B] .
$$

This proves the lemma.

The same method proves the following lemma that will be useful later. Let $\rho^{\prime}: X^{\prime} \rightarrow \mathcal{M}$ be a $\sigma$-regularizing morphism; let $\tilde{\sigma}^{\prime}: \tilde{E}^{\prime}=\rho^{\prime *} E \rightarrow \mathcal{O}_{X^{\prime}}\left(D^{\prime}\right)$, $\tilde{G}^{\prime}=\operatorname{ker}\left\{\tilde{\sigma}^{\prime}\right\}$, and $\rho^{\prime}(\sigma):\left|D^{\prime}\right| \rightarrow \mathcal{M}(\sigma)$ be the tautological projection, as in the proof of Lemma 2.5.

Lemma 2.7. Let the notation be as earlier. Suppose $\left[\tilde{B}^{\prime}\right] \in Z_{d} \tilde{G}^{\prime}$ such that $\tilde{\rho}_{*}^{\prime}\left[\tilde{B}^{\prime}\right]=$ $0 \in Z_{d} E$. Then $\rho^{\prime}(\sigma)_{*}\left(\left[D^{\prime}\right] \cdot s_{\tilde{G}^{\prime}}^{!}\left(\left[\tilde{B}^{\prime}\right]\right)\right)=0 \in A_{d-r} \mathcal{M}(\sigma)$.

Proof. We let $B=\tilde{\rho}^{\prime}\left(\tilde{B}^{\prime}\right)$. Since $\tilde{\rho}^{\prime}$ is proper, $B$ is closed and integral in $E$; since $\tilde{\rho}_{*}^{\prime}\left(\left[\tilde{B}^{\prime}\right]\right)=0$ in $Z_{d} E, \operatorname{dim} B<d$. We let $B_{0}=\pi(B) \subset \mathcal{M}$. We then pick $\rho: X \rightarrow \mathcal{M}$ a $\sigma$-regularizing morphism so that $\rho(X)=B_{0}$ and $\rho: X \rightarrow B_{0}$ is generically finite.

We form $Y=X \times_{\mathcal{M}} X^{\prime}$ and let $p: Y \rightarrow \mathcal{M}$ be the induced morphism. As in the proof of Lemma 2.5 (and using the notation developed in the proof), we have $\bar{E}=p^{*} E \rightarrow Y, \tilde{q}^{\prime}: \bar{E} \rightarrow \tilde{E}^{\prime}$, and an integral $\bar{B} \subset \bar{E}$ so that $\tilde{q}^{\prime}(\bar{B})=\tilde{B}^{\prime}$. Let $\tilde{q}: \bar{E} \rightarrow \tilde{E}$ and let $\tilde{B}=\tilde{q}(\bar{B})$. Since $\tilde{B}$ is integral, since $\tilde{\rho}(\tilde{B})=B$, since $B \rightarrow B_{0}$ is dominant, and since $\rho: X \rightarrow B_{0}$ is dominant and generically finite, $\operatorname{dim} \tilde{B}=\operatorname{dim} \tilde{\rho}(\tilde{B})=\operatorname{dim} B_{0}<d$. Thus $\tilde{q}_{*}([\bar{B}])=0 \in Z_{d} \tilde{E}$. Therefore,

$$
k \cdot \rho^{\prime}(\sigma)_{*}\left(\left[D^{\prime}\right] \cdot s_{\tilde{G}^{\prime}}^{!}\left(\left[\tilde{B}^{\prime}\right]\right)\right)=p(\sigma)_{*}\left([\bar{D}] \cdot s_{\bar{G}}^{!}([\bar{B}])\right)=\rho(\sigma)_{*}\left([D] \cdot s_{\tilde{G}} \tilde{\sigma}\left(\left[\tilde{q}_{*} \bar{B}\right]\right)\right)=0 .
$$

This proves the lemma.

We next show that the map $s_{E, \sigma}^{!}$preserves rational equivalence. In this paper, we adopt the the convention that a rational equivalence $R \in W_{d} E$ is a sum $R=$ $\sum_{\alpha \in \Lambda} n_{\alpha}\left[S_{\alpha}, \phi_{\alpha}\right]$, where $S_{\alpha} \subset E$ are $(d+1)$-dimensional closed integrals and $\phi_{\alpha} \in$ $\mathbb{k}\left(S_{\alpha}\right)^{*}$. We also use $\partial_{0}[S, \phi]=(\phi=0) \cdot[S]$ and $\partial_{\infty}[S, \phi]=(\phi=\infty) \cdot[S]$.

Lemma 2.8. For $R \in W_{d} E(\sigma), s_{E, \sigma}^{!}\left(\partial_{0} R\right)=s_{E, \sigma}^{!}\left(\partial_{\infty} R\right)$.

Proof. We only need to prove the lemma for $R=[S, \phi] \in W_{d} E(\sigma)$, where $S \subset$ $E(\sigma)$ is closed and integral. In case $\left.S \subset E\right|_{\mathcal{M}(\sigma)}$, then both $\partial_{0} R$ and $\partial_{\infty} R \in$ $\left.Z_{d} E\right|_{\mathcal{M}(\sigma)}$, and the required identity holds because the usual Gysin map $s_{\left.E\right|_{\mathcal{M}(\sigma)}}$ : $Z_{d}\left(\left.E\right|_{\mathcal{M}(\sigma)}\right) \rightarrow A_{d-r} \mathcal{M}(\sigma)$ preserves the rational equivalence.

We now suppose $\left.S \not \subset E\right|_{\mathcal{M}(\sigma)}$. We let $S_{0}=\pi(S) \subset \mathcal{M}$. We pick a proper and generically finite $\sigma$-regularizing $\rho: X \rightarrow \mathcal{M}$ so that $\rho(X)=S_{0}$. We let $\tilde{\sigma}: \tilde{E}:=\rho^{*} E \rightarrow \mathcal{O}_{X}(D)$ be the surjective homomorphism extending $\rho^{*} \sigma$. By Lemma 2.5, such a $\rho$ exists. Because $\rho$ is generically finite, we can find a closed integral $(d+1)$-dimensional $\tilde{S} \subset \tilde{E}$ so that with $\tilde{\rho}: \tilde{E} \rightarrow E$ the projection, $\tilde{\rho}(\tilde{S})=S$.

We let $\tilde{G}=\operatorname{ker}\left\{\tilde{\sigma}: \tilde{E} \rightarrow \mathcal{O}_{X}(D)\right\}$. Because $S \subset E(\sigma)$ and $\left.S \not \subset E\right|_{\mathcal{M}(\sigma)}, \tilde{S} \subset \tilde{G}$. We let $\tilde{\phi}$ be the pull-back $\tilde{\rho}^{*} \phi$ and let $\tilde{R}=[\tilde{S}, \tilde{\phi}]$. Thus, $\tilde{\rho}_{*} \tilde{R} \in W_{d} E(\sigma)$ and $\tilde{\rho}_{*} \tilde{R}=k \cdot R$, for an integer $k$; since $\tilde{\rho}$ is proper,

$$
\tilde{\rho}_{*}\left(\partial_{0} \tilde{R}\right)=k \cdot \partial_{0} R \quad \text { and } \quad \tilde{\rho}_{*}\left(\partial_{\infty} \tilde{R}\right)=k \cdot \partial_{\infty} R .
$$

We now decompose the cycle $\partial_{0} R=B_{1}+B_{2}$ so that each summand $[T] \in B_{1}$ (resp. $[T] \in B_{2}$ ) satisfies $\left.T \subset E\right|_{\mathcal{M}(\sigma)}$ (resp. $\left.T \not \subset E\right|_{\mathcal{M}(\sigma)}$ ). We also decompose $\partial_{\infty} R=C_{1}+C_{2}$ according to the same rule with $B_{i}$ replaced by $C_{i}$. 
We decompose $\partial_{0} \tilde{R}$ into the sum of three parts, $\partial_{0} \tilde{R}=\tilde{B}_{0}+\tilde{B}_{1}+\tilde{B}_{2}$, so that $[T] \in \tilde{B}_{0}$ (resp. $[T] \in \tilde{B}_{1}$; resp. $[T] \in \tilde{B}_{2}$ ) satisfying $\tilde{\rho}_{*}([T])=0$ (resp. $\left.T \subset \tilde{E}\right|_{|D|}$; resp. $\left.\left.T \not \subset \tilde{E}\right|_{|D|}\right)$. By moving all summands $[T]$ with $\tilde{\rho}_{*}[T]=0$ to $\tilde{B}_{0}$, no $[T]$ appears simultaneously in two of the three factors $\tilde{B}_{0}, \tilde{B}_{1}$, and $\tilde{B}_{2}$. We decompose $\partial_{\infty} \tilde{R}=\tilde{C}_{0}+\tilde{C}_{1}+\tilde{C}_{2}$ according to the same rule with $\tilde{B}_{i}$ replaced by $\tilde{C}_{i}$. By (2.4), and using the property of the decompositions, we have $\tilde{\rho}_{*}\left(\tilde{B}_{i}\right)=k \cdot B_{i}$ and $\tilde{\rho}_{*}\left(\tilde{C}_{i}\right)=k \cdot C_{i}$ for $i=1,2$.

Applying the definition of $s_{E, \sigma}^{!}$to $\partial_{0} R$ (resp. $\partial_{\infty} R$ ):

$$
s_{E, \sigma}^{!}\left(\partial_{0} R\right)=s_{\left.E\right|_{\mathcal{M}(\sigma)}}\left(B_{1}\right)+k^{-1} \rho(\sigma)_{*}\left([D] \cdot s_{\tilde{G}}^{!}\left(\tilde{B}_{2}\right)\right)
$$

(resp. the same formula with $\partial_{0} R$ replaced by $\partial_{\infty} R$ ), we obtain identities in $A_{d-r} \mathcal{M}(\sigma)$ :

$$
s_{E, \sigma}^{!}\left(\partial_{0} R\right)-s_{E, \sigma}^{!}\left(\partial_{\infty} R\right)=s_{\left.E\right|_{\mathcal{M}(\sigma)}}^{!}\left(B_{1}-C_{1}\right)+k^{-1} \cdot \rho(\sigma)_{*}\left([D] \cdot s_{\tilde{G}^{\prime}}^{!}\left(\tilde{B}_{2}-\tilde{C}_{2}\right)\right) .
$$

We claim that

$$
\rho(\sigma)_{*}\left([D] \cdot s_{\tilde{G}}^{!}\left(\partial_{0} \tilde{R}-\partial_{\infty} \tilde{R}\right)\right)=0 \in A_{d-r} \mathcal{M}(\sigma) .
$$

This is true because $s_{\tilde{G}}^{!}\left(\partial_{0} \tilde{R}-\partial_{\infty} \tilde{R}\right)=0 \in A_{d-r+1} X$, and $\rho(\sigma)_{*}$ and $[D]$. preserve rational equivalence.

Applying Lemma 2.7, we also have

$$
\rho(\sigma)_{*}\left([D] \cdot s_{\tilde{G}}^{!}\left(\tilde{B}_{0}\right)\right)=\rho(\sigma)_{*}\left([D] \cdot s_{\tilde{G}}^{!}\left(\tilde{C}_{0}\right)\right)=0 \in A_{d-r} \mathcal{M}(\sigma) .
$$

Furthermore, because of Lemma 2.6 and the fact that $\tilde{B}_{1}$ and $\tilde{C}_{1}$ lie over $|D|$,

$$
\rho(\sigma)_{*}\left([D] \cdot s_{\tilde{G}}^{!}\left(\tilde{B}_{1}-\tilde{C}_{1}\right)\right)=\rho(\sigma)_{*} s_{\left.\tilde{E}\right|_{|D|}}\left(\tilde{B}_{1}-\tilde{C}_{1}\right)=s_{\left.E\right|_{\mathcal{M}(\sigma)}}^{!}\left(\tilde{\rho}_{*} \tilde{B}_{1}-\tilde{\rho}_{*} \tilde{C}_{1}\right) .
$$

Therefore, using $\tilde{B}_{2}-\tilde{C}_{2}=\left(\partial_{0} \tilde{R}-\partial_{\infty} \tilde{R}\right)-\left(\tilde{B}_{0}-\tilde{C}_{0}\right)-\left(\tilde{B}_{1}-\tilde{C}_{1}\right)$, (2.5) and (2.6), and $\tilde{\rho}_{*}\left(\tilde{B}_{1}\right)=k \cdot B_{1}$ and same for $C_{1}$, we obtain $s_{E, \sigma}^{!}\left(\partial_{0} R\right)-s_{E, \sigma}^{!}\left(\partial_{\infty} R\right)=0$. This proves the lemma.

Corollary 2.9. The basic construction defines a homomorphism

$$
s_{E, \sigma}^{!}: A_{*} E(\sigma) \rightarrow A_{*-r} \mathcal{M}(\sigma),
$$

which we call the localized Gysin map. Furthermore, if we let $\iota: \mathcal{M}(\sigma) \rightarrow \mathcal{M}$ and $\tilde{\iota}: E(\sigma) \rightarrow E$ be the inclusions, then $\iota_{*} \circ s_{E, \sigma}^{!}=s_{E}^{!} \circ \tilde{\iota}_{*}: A_{*} E(\sigma) \rightarrow A_{*-r} \mathcal{M}$.

Proof. The first part is the combination of Lemmas 2.8 and 2.5. The second part is the consequence of Lemma 2.6.

\section{LOCALIZED GySin MAPS FOR BUNDLE STACKS}

To construct localized virtual cycles, we need to generalize the localized Gysin map to bundle stacks over a DM stack $\mathcal{M}$.

Let $E^{\bullet} \in D(\mathcal{M})$ be a derived (category) object that is locally quasi-isomorphic to a two-term complex of locally free sheaves concentrated at $[0,1]$. We let $\mathbf{E}=$ $h^{1} / h^{0}\left(E^{\bullet}\right)$, which is a bundle stack isomorphic to $\left[E^{1} / E^{0}\right]$ in case $E^{\bullet} \cong_{q i s}\left[E^{0} \rightarrow\right.$ $E^{1}$ ] (cf. [2]).

In case there is a vector bundle $V$ that surjects onto $\mathbf{E}$, one defines $s_{\mathbf{E}}^{!}: A_{*} \mathbf{E} \rightarrow$ $A_{*} \mathcal{M}$ as the composite of the flat pull-back $A_{*} \mathbf{E} \rightarrow A_{*} V$ and the Gysin map $s_{V}^{!}: A_{*} V \rightarrow A_{*} \mathcal{M}$. 
Without such a vector bundle, one can either use the intersection theory developed by Kresch [16] to define $s_{\mathbf{E}}^{!}$or follow the recipe developed in 25] by the second author of this paper. We will follow the latter approach in this paper.

We suppose there is a surjective homomorphism of sheaves on an open substack $U \subset \mathcal{M}$

$$
\sigma:\left.h^{1}\left(E^{\bullet}\right)\right|_{U} \longrightarrow \mathcal{O}_{U}
$$

It induces a morphism from the bundle stack $\left.\mathbf{E}\right|_{U}$ to the line bundle $\mathbb{C}_{U}$. As earlier, we let $\mathcal{M}(\sigma)=\mathcal{M} \backslash U$. We let $\mathbf{E}(\sigma)$ be the kernel cone stack in $\mathbf{E}$,

$$
\mathbf{E}(\sigma)=\left.\mathbf{E}\right|_{\mathcal{M}(\sigma)} \cup \operatorname{ker}\left\{\left.\mathbf{E}\right|_{U} \rightarrow \mathbb{C}_{U}\right\} \subset \mathbf{E}
$$

endowed with the reduced structure. (Since $\sigma$ is surjective on $U,\left.\mathbf{E}\right|_{U} \rightarrow \mathbb{C}_{U}$ is surjective; thus the kernel is well-defined and is a closed substack of $\left.\mathbf{E}\right|_{U}$.)

Our goal is to construct a localized Gysin map

$$
s_{\mathbf{E}, \sigma}^{!}: A_{*} \mathbf{E}(\sigma) \longrightarrow A_{*} \mathcal{M}(\sigma) .
$$

It is constructed by finding for each irreducible cycle $[\mathbf{c}] \in Z_{*} \mathbf{E}(\sigma)$ a proper representative $m_{X}^{-1}[C] \in Z_{*} F$ of $[\mathbf{c}]$ in a vector bundle $F$ over a variety $X$ with a surjective meromorphic cosection $\left(F, \sigma_{X}\right)$ and a proper $\rho: X \rightarrow \mathcal{M}$ and then defining

$$
s_{\mathbf{E}, \sigma}^{!}([\mathbf{c}])=m_{X}^{-1} \rho(\sigma)_{*}\left(s_{F, \sigma_{X}}^{!}([C])\right) \in A_{*} \mathcal{M}(\sigma) .
$$

We remark here that any homomorphism $V \rightarrow h^{1}\left(E^{\bullet}\right)$ from a locally free sheaf $V$ to $h^{1}\left(E^{\bullet}\right)$ induces canonically a bundle-stack morphism $V \rightarrow \mathbf{E}$. Indeed, let $\eta: M \rightarrow \mathcal{M}$ be an étale open covering so that $\eta^{*} E^{\bullet} \cong_{q i s}\left[F^{0} \rightarrow F^{1}\right]$ with both $F^{i}$ locally free on $M$. We lift the pull-back $\eta^{*} V \rightarrow \eta^{*} h^{1}\left(E^{\bullet}\right)$ to a homomorphism $\eta^{*} V \rightarrow F^{1}$, which defines a homomorphism of complexes $\eta^{*} V[-1] \rightarrow\left[F^{0} \rightarrow F^{1}\right]$. By the functorial construction of $h^{1} / h^{0}$, we obtain a morphism

$$
\eta^{*} V=h^{1} / h^{0}\left(\eta^{*} V[-1]\right) \rightarrow h^{1} / h^{0}\left(\eta^{*} E^{\bullet}\right)=\mathbf{E} \times{ }_{\mathcal{M}} M .
$$

One checks that any two liftings $\eta^{*} V \rightarrow F^{1}$ define homotopic $\eta^{*} V[-1] \rightarrow\left[F^{0} \rightarrow\right.$ $\left.F^{1}\right]$, and thus induce canonically isomorphic maps $\eta^{*} V \rightarrow \mathbf{E} \times \mathcal{M} M$. Since the morphism so constructed is canonical, it descends to a morphism

$$
V \longrightarrow \mathbf{E} \text {. }
$$

We now construct representatives of irreducible $[\mathbf{c}] \in Z_{*} \mathbf{E}$. Let $\pi: \mathbf{E} \rightarrow \mathcal{M}$ be the projection; let $M_{\mathbf{c}}$ be the closure of the image $\pi(\mathbf{c}) \subset \mathcal{M}$. Applying Chow's Lemma [18, Cor. 16.6.1], we can find a quasi-projective variety $X$, a proper and surjective morphism $\rho: X \rightarrow M_{\mathbf{c}}$ such that for a Zariski dense open subset $X_{0} \subset X$, $\left.\rho\right|_{X_{0}}: X_{0} \rightarrow M_{\mathbf{c}}$ is étale. Since $X$ is quasi-projective, we can find a complex of locally free sheaves $F^{\bullet}=\left[F_{0} \rightarrow F_{1}\right]$ of $\mathcal{O}_{X}$-modules that is quasi-isomorphic to $\rho^{*} E^{\bullet}$. By abuse of notation, we view $F_{1}$ as a vector bundle over $X$ and obtain a flat morphism

$$
\gamma: F_{1} \longrightarrow h^{1} / h^{0}\left(F^{\bullet}\right)=h^{1} / h^{0}\left(\rho^{*} E^{\bullet}\right)=\rho^{*} \mathbf{E} .
$$

Since $\left.\rho\right|_{X_{0}}: X_{0} \rightarrow M_{\mathbf{c}}$ is étale, the induced

$$
\left.\gamma\right|_{X_{0}}: F_{1} \times\left._{X} X_{0} \longrightarrow \mathbf{E}\right|_{M_{\mathbf{c}}}=h^{1} / h^{0}\left(E^{\bullet}\right) \times_{\mathcal{M}} M_{\mathbf{c}}
$$

is flat. 
Definition 3.1. A proper representative of an irreducible $[\mathbf{c}] \in Z_{*} \mathbf{E}$ consists of $\left(\rho, F_{1}\right)$ just constructed and a cycle $C_{X}=\sum_{i} m_{i}\left[C_{i}\right] \in Z_{*} F_{1}$, where $C_{i}$ are closed and integral in $F_{1}$ and $m_{i} \in \mathbb{Z}$, such that $\sum_{i} m_{i}\left[C_{i} \times_{X} X_{0}\right]=\left(\left.\gamma\right|_{X_{0}}\right)^{*}(\mathbf{c})$, and $C_{i}=\overline{C_{i} \times{ }_{X} X_{0}}$ for all $i$.

Using proper representatives of $[\mathbf{c}] \in Z_{*} \mathbf{E}$, we can define $s_{\mathbf{E}, \sigma}^{!}$at the cycle level. Let $[\mathbf{c}] \in Z_{*} \mathbf{E}(\sigma)$ be an irreducible cycle. Let $\left(\rho, X, F_{1}\right)$ with $\sum_{i} m_{i} C_{i}$ be a proper representative of $[\mathbf{c}]$. Let $\sigma_{X}:\left.F_{1}\right|_{\rho^{-1} U} \rightarrow \mathcal{O}_{\rho^{-1} U}$ be the composite of $\left.F_{1}\right|_{\rho^{-1} U} \rightarrow$ $\left.\rho^{*} h^{1}\left(E^{\bullet}\right)\right|_{\rho^{-1} U}$ with $\rho^{*} \sigma$. Automatically $\left[C_{i}\right] \in Z_{*} F_{1}\left(\sigma_{X}\right)$. We let $\rho(\sigma): X\left(\sigma_{X}\right) \rightarrow$ $\mathcal{M}(\sigma)$ be the restricton of $\rho$ to $X\left(\sigma_{X}\right)=X \backslash \rho^{-1} U$.

Definition 3.2. We define $s_{\mathbf{E}, \sigma}^{!}([\mathbf{c}])=m_{X}^{-1} \rho(\sigma)_{*}\left(s_{F_{1}, \sigma_{X}}^{!}\left(\left[C_{X}\right]\right)\right)$, where $m_{X}$ is the degree of $\rho: X \rightarrow M_{\mathbf{c}}$. We extend it to $s_{\mathbf{E}, \sigma}: Z_{*} \mathbf{E} \rightarrow A_{*} \mathcal{M}(\sigma)$ by linearity.

Proposition 3.3. The map $s_{\mathbf{E}, \sigma}$ in Definition 3.2 is well-defined. The map $s_{\mathbf{E}, \sigma}$ preserves the rational equivalence and thus defines a (localized Gysin) homomoprhism

$$
s_{\mathbf{E}, \sigma}^{!}: A_{*} \mathbf{E}(\sigma) \longrightarrow A_{*} \mathcal{M}(\sigma) .
$$

Proof. Since the existence of proper representatives of $[\mathbf{c}]$ is guaranteed by the Chow Lemma, to show that the localized Gysin map is well-defined at the cycle level we need to check that it is independent of the choice of proper representatives.

To check that it is well-defined, we need to show that if $m_{X^{\prime}}^{-1}\left[C_{X^{\prime}}\right]$ with $\left(\rho^{\prime}, X^{\prime}, F_{1}^{\prime}\right)$ is another proper representative of $[\mathbf{c}]$, then

$$
m_{X}^{-1} \rho(\sigma)_{*}\left(s_{F_{1}, \sigma_{X}}^{!}\left(\left[C_{X}\right]\right)\right)=m_{X^{\prime}}^{-1} \rho^{\prime}(\sigma)_{*}\left(s_{F_{1}^{\prime}, \sigma_{X^{\prime}}}^{!}\left(\left[C_{X^{\prime}}\right]\right)\right) .
$$

This can be proved by choosing a third proper representative using $Y \subset X \times{ }_{\mathcal{M}} X^{\prime} \rightarrow$ $\mathcal{M}$ an irreducible component, choosing a complex $\bar{F}^{\bullet}$ quasi-isomorphic to the pullbacks of $F^{\bullet}$ and $\left(F^{\prime}\right)^{\bullet}$ and whose projections commute with the projections to the pull-backs of $h^{1}\left(E^{\bullet}\right)$. Since $X \rightarrow M_{\mathbf{c}}$ and $X^{\prime} \rightarrow M_{\mathbf{c}}$ are étale over dense open subsets, we can choose $Y$ so that $Y \rightarrow M_{\mathbf{c}}$ is étale over a dense open subset as well. The remaining proof is parallel to the proof of Lemma 2.5] and will be omitted. This defines the homomorphism $s_{\mathbf{E}, \sigma}^{!}: Z_{*} \mathbf{E}(\sigma) \longrightarrow A_{*} \mathcal{M}(\sigma)$.

Finally, we check that it preserves the rational equivalence. This time the proof is a line by line repetition of the proof of Lemma 2.8, incorporating the need to use proper representatives to make sense of degrees of maps. Since the modification is routine, we will omit the details here.

\section{REDUCING INTRINSIC NORMAL CONES BY COSECTIONS}

In this section, we show that a Deligne-Mumford stack equipped with a perfect obstruction theory and a meromorphic cosection of its obstruction sheaf has "restricted" intrinsic normal cones. Applying the localized Gysin maps, we obtain the localized virtual cycles.

We let $\pi: \mathcal{M} \rightarrow \mathcal{S}$ be a DM stack $\mathcal{M}$ over a smooth Artin stack $\mathcal{S}$; we assume $\pi$ is representable. We assume $\mathcal{M} / \mathcal{S}$ admits a relative perfect obstruction theory 
$E^{\bullet} \rightarrow L_{\mathcal{M} / \mathcal{S}}$ formulated in [2] using cotangent complexes 1 As part of the definition, locally $E^{\bullet}$ is quasi-isomorphic to two-term complexes of locally free sheaves concentrated at $[-1,0]$.

We introduce the obstruction sheaf of a relative perfect obstruction theory. We recall that $\mathcal{O} b_{\mathcal{M} / \mathcal{S}}=h^{1}\left(\left(E^{\bullet}\right)^{\vee}\right)$ is the relative obstruction sheaf of $E^{\bullet} \rightarrow L_{\mathcal{M} / \mathcal{S}}^{\bullet}$. To introduce its (absolute) obstruction sheaf, we pick a smooth chart $M / S$ of $\mathcal{M} / \mathcal{S}$ by affine schemes $M$ and $S$ such that $S \rightarrow \mathcal{S}$ is smooth, $M \subset \mathcal{M} \times \mathcal{S} S$ is open, and $M \rightarrow \mathcal{M}$ is étale. (This is possible since $\mathcal{M} \rightarrow \mathcal{S}$ is representable.) We pick an $S$-embedding $M \rightarrow V$ into an affine $V$, smooth over $S$. Since $M$ is affine, we pick a presentation $\left.E^{\bullet}\right|_{M}=\left[E^{-1} \rightarrow E^{0}\right]$ as a complex of locally free sheaves so that the perfect obstruction theory of $\mathcal{M} / \mathcal{S}$ lifted to $M / S$ is given by a homomorphism of complexes of sheaves

$$
\left(\phi^{-1}, \phi^{0}\right):\left[E^{-1} \rightarrow E^{0}\right] \rightarrow \tau^{\geq-1} L_{M / S}^{\bullet}=\left[I_{M} /\left.I_{M}^{2} \rightarrow \Omega_{V / S}\right|_{M}\right],
$$

where $I_{M}$ is the ideal sheaf of $M \subset V$. We let $\pi_{S}: M \rightarrow S$ be the projection.

We denote

$$
\mathcal{O} b_{M / S}:=\mathcal{O} b_{\mathcal{M} / \mathcal{S}} \otimes_{\mathcal{O}_{\mathcal{M}}} \mathcal{O}_{M}=h^{1}\left(\left(\left.E^{\bullet}\right|_{M}\right)^{\vee}\right) .
$$

From the distinguished triangle

$$
\pi_{S}^{*} L_{S}^{\bullet} \longrightarrow L_{M}^{\bullet} \longrightarrow L_{M / S}^{\bullet} \longrightarrow \pi_{S}^{*} L_{S}^{\bullet}[1]=\left[\pi_{S}^{*} \Omega_{S} \rightarrow 0\right],
$$

we have the composition

$$
E^{\bullet} \longrightarrow \tau^{\geq-1} L_{M / S}^{\bullet} \longrightarrow \pi_{S}^{*} \Omega_{S}[1]
$$

and a distinguished triangle

$$
\hat{E}^{\bullet} \longrightarrow E^{\bullet} \longrightarrow \pi_{S}^{*} \Omega_{S}[1],
$$

which fits into a commutative diagram of distinguished triangles

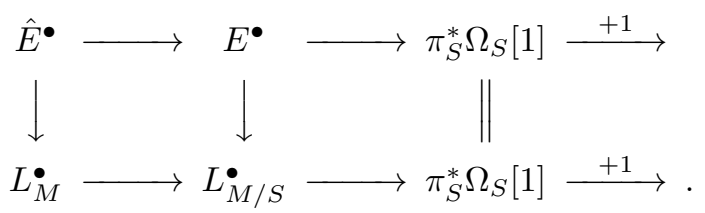

By the standard 5-lemma, we find that $\hat{E}^{\bullet} \rightarrow L_{M}^{\bullet}$ is a perfect obstruction theory of $M$ and that the obstruction sheaf $\mathcal{O} b_{M}$ is the quotient

$$
\mathcal{O} b_{M / S} \longrightarrow \mathcal{O} b_{M}=\operatorname{coker}\left\{\pi_{S}^{*} \Omega_{S}^{\vee} \longrightarrow \mathcal{O} b_{M / S}\right\}
$$

of $\mathcal{O} b_{M / S}$.

Since this construction is canonical, the object $\hat{E}^{\bullet}$ is unique up to quasi-isomorphism; the arrow $\pi_{S}^{*} \Omega_{S} \rightarrow \hat{E}^{\bullet}$ (in (4.2) ) is unique up to homotopy. Thus $\mathcal{O} b_{M}=$

\footnotetext{
${ }^{1}$ We recall that there are two versions of perfect obstruction theories: one formulated by Behrend-Fantechi (BF for short) [2] using an arrow from a derived object to the relative cotangent complex $E^{\bullet} \rightarrow \mathrm{E}_{\mathcal{M} / \mathcal{S}}$; the other by Li-Tian (LT for short) 24] using obstruction to deformation assignment in the cohomology of a derived object. By [2, Theorem 4.5], the BF version of perfect obstruction theories induces LT's version of perfect obstruction theories.

Conversely, it will be shown in [7] that LT's version of perfect obstruction theory is affine locally equivalent to BF's version. Furthermore, all available technical tools concerning cycles constructed from BF's version of perfect obstruction theory work for LT's version as well.
} 
$h^{1}\left(\left(\hat{E}^{\bullet}\right)^{\vee}\right)$ in (4.3) is canonically defined, and the first arrow in (4.3) is unique. This proves that (4.3) descends to a quotient homomorphism of sheaves on $\mathcal{M}$

$$
\mathcal{O} b_{\mathcal{M} / \mathcal{S}} \longrightarrow \mathcal{O} b_{\mathcal{M}}
$$

Definition 4.1. We call $\mathcal{O} b_{\mathcal{M}}$ the (absolute) obstruction sheaf of the obstruction theory $E^{\bullet} \rightarrow L_{\mathcal{M} / \mathcal{S}}^{\bullet}$

We denote

$$
\mathbf{E}:=h^{1} / h^{0}\left(\left(E^{\bullet}\right)^{\vee}\right)
$$

and let $\mathbf{c}_{\mathcal{M}} \subset \mathbf{E}$ be the relative intrinsic normal cone introduced in [2]. A meromorphic cosection of $\mathcal{O} b_{\mathcal{M}}$ will reduce the intrinsic normal cone $\left[\mathbf{c}_{\mathcal{M}}\right]$ to a subcone-stack of $\mathbf{E}$. Let $U \subset \mathcal{M}$ be an open substack and let

$$
\sigma:\left.\mathcal{O} b_{\mathcal{M}}\right|_{U} \longrightarrow \mathcal{O}_{U}
$$

be a surjective homomorphism. As earlier, we call $\sigma$ a meromorphic cosection surjective on $U$; we call $\mathcal{M}(\sigma)=\mathcal{M}-U$ the degeneracy locus of $\sigma$.

The homomorphism $\sigma$ induces a homomorphism $\left.\left(E^{\bullet}\right)^{\vee}\right|_{U} \rightarrow\left[0 \rightarrow \mathcal{O}_{U}\right]$, and thus a surjective cone-stack morphism $\bar{\sigma}:\left.\mathbf{E}\right|_{U} \longrightarrow h^{1} / h^{0}\left(\left[0 \rightarrow \mathcal{O}_{U}\right]\right)=\mathbb{C}_{U}$. (Here we use $\mathbb{C}_{\mathcal{M}}$ to denote the trivial line bundle $\mathbb{A}^{1} \times \mathcal{M} \rightarrow \mathcal{M}$.)

Definition 4.2. We define $\mathbf{E}(\sigma)$ to be the union of $\mathbf{E} \times \mathcal{M} \mathcal{M}(\sigma)$ with $\operatorname{ker}\{\bar{\sigma}:$ $\left.\left.\mathbf{E}\right|_{U} \rightarrow \mathbb{C}_{U}\right\}$, endowed with the reduced structure. The closed substack $\mathbf{E}(\sigma) \subset \mathbf{E}$ is called the kernel cone-stack of $\sigma$.

Proposition 4.3. Let the notation be as stated, and let $\sigma:\left.\mathcal{O} b_{\mathcal{M}}\right|_{U} \rightarrow \mathcal{O}_{U}$ be surjective. Then the cycle $\left[\mathbf{c}_{\mathcal{M}}\right] \in Z_{*} \mathbf{E}$ lies in $Z_{*} \mathbf{E}(\sigma)$.

We consider a simple case. Let $M \subset V$ be a closed subscheme of a smooth scheme $V$ defined by the vanishing $s=0$ of a section $s$ of a vector bundle $E$ on $V$; let $C_{M / V}$ be the normal cone to $M$ in $V$, embedded in $\left.E\right|_{M}$ via the section $s$. We suppose $\bar{\sigma}:\left.E\right|_{M} \rightarrow \mathcal{O}_{M}$ is a surjective homomorphism. Let $I_{M}$ be the ideal sheaf of $M \subset V$.

Lemma 4.4 (Cone reduction criterion). Suppose the defining equation s satisfies the following criterion: for any germ $\varphi: \operatorname{Spec} \mathbb{k} \llbracket \xi \rrbracket \rightarrow V, \varphi(0) \in M$, the section $\bar{\sigma} \circ s \circ \varphi \in \mathbb{k} \llbracket \xi \rrbracket$ satisfies $\bar{\sigma} \circ s \circ \varphi \in \xi \cdot \varphi^{*} I_{M}$. Then the support of the cone $C_{M / V}$ lies entirely in the kernel $F=\operatorname{ker}\left\{\bar{\sigma}:\left.E\right|_{M} \rightarrow \mathcal{O}_{M}\right\} \subset E$.

Proof. The cone $C_{M / V} \subset E$ is the specialization of the section $t^{-1} s \subset E$ as $t \rightarrow 0$. More precisely, we consider the subscheme

$$
\Gamma=\left\{\left(t^{-1} s(w), t\right) \in E \times\left(\mathbb{A}^{1} \backslash 0\right) \mid w \in V, t \in \mathbb{A}^{1} \backslash 0\right\} .
$$

For $t \in \mathbb{A}^{1} \backslash 0$, the fiber $\Gamma_{t}$ of $\Gamma$ over $t \in \mathbb{A}^{1}$ is the section $t^{-1} s$ of $E$. We let $\bar{\Gamma}$ be the closure of $\Gamma$ in $E \times \mathbb{A}^{1}$. The central fiber $\bar{\Gamma} \times_{\mathbb{A}^{1}} 0 \subset E$ is the normal cone $C_{M / V}$ embedded in $\left.E\right|_{M}$. Clearly, $C_{M / V}$ is of pure dimension $\operatorname{dim} V$.

Now let $N \subset C_{M / V}$ be any irreducible component. Let $v \in N$ be a general closed point of $N$. Then we can find a smooth affine curve $0 \in S$ and a morphism $\rho:(0, S) \rightarrow(v, \bar{\Gamma})$ such that $\rho(S \backslash 0) \subset \Gamma$. We let $\rho_{V}: S \rightarrow V$ and $\rho_{\mathbb{A}^{1}}: S \rightarrow \mathbb{A}^{1}$ be the composites of $\rho$ with the projections from $E \times \mathbb{A}^{1}$ to $V$ and to $\mathbb{A}^{1}$. Since $\rho(S \backslash 0) \subset \Gamma, \rho_{\mathbb{A}^{1}}$ dominates $\mathbb{A}^{1}$. 
We then choose a uniformizing parameter $\xi$ of $S$ at 0 so that $\left(\rho_{\mathbb{A}^{1}}\right)^{*}(t)=\xi^{n}$ for some $n$. Because $\rho(0)=v, \xi^{-n} \cdot s \circ \rho_{V}: S \backslash 0 \rightarrow E$ specializes to $v$; hence $s \circ \rho_{V}$ has the expression

$$
s \circ \rho_{V}=v \xi^{n}+O\left(\xi^{n+1}\right) .
$$

Applying $\bar{\sigma}$, we obtain $\bar{\sigma} \circ s \circ \rho_{V}=\bar{\sigma}(v) \xi^{n}+O\left(\xi^{n+1}\right)$.

Now suppose $N \not \subset E(\sigma)$; in particular $v$ does not lie in the zero section of $E$. Then $\rho_{V}^{*} I_{M}=\left(\xi^{n}\right)$. By assumption, $\bar{\sigma} \circ s \circ \rho_{V} \in\left(\xi^{n+1}\right)=\xi \cdot \rho_{V}^{*} I_{M}$. We must have $\bar{\sigma}(v)=0$. This proves that $v \in F=\operatorname{ker}\{\bar{\sigma}\}$. Since $v$ is general in $C_{M / V}, v \in F$ implies that the support of $C_{M / V}$ lies in $F$. This proves the lemma.

Let $\mathcal{O} b_{M}=\operatorname{coker}\left\{d s:\left.\left.T_{V}\right|_{M} \rightarrow E\right|_{M}\right\}$; let pr: $\left.E\right|_{M} \rightarrow \mathcal{O} b_{M}$ be the projection.

Corollary 4.5. Let the notation be as in Lemma 4.4. Suppose we have a surjective homomorphism $\sigma: \mathcal{O b}_{M} \rightarrow \mathcal{O}_{M}$. Then the support of the cone $C_{M / V}$ lies entirely in the kernel $F=\operatorname{ker}\left\{\sigma \circ \mathrm{pr}:\left.E\right|_{M} \rightarrow \mathcal{O}_{M}\right\} \subset E$.

Proof. We verify the cone reduction criterion. Let $\varphi: \operatorname{Spec} \mathbb{k} \llbracket \xi \rrbracket \rightarrow V, \varphi(0) \in M$, be any morphism. Suppose $\varphi^{*} I_{M}=\left(\xi^{n}\right)$. Pulling back the exact sequence

$$
\mathcal{O}_{M}\left(T_{V}\right) \stackrel{d s}{\longrightarrow} \mathcal{O}_{M}(E) \longrightarrow \mathcal{O} b_{M} \longrightarrow 0
$$

via the induced morphism $\bar{\varphi}=\left.\varphi\right|_{\varphi^{-1}(M)}: \varphi^{-1}(M)=\left(\xi^{n}=0\right) \rightarrow M$, we obtain

$$
\bar{\varphi}^{*}\left(T_{V}\right) \stackrel{\bar{\varphi}^{*}(d s)}{\longrightarrow} \bar{\varphi}^{*}(E) \stackrel{\bar{\varphi}^{*}(\mathrm{pr})}{\longrightarrow} \bar{\varphi}^{*}\left(\mathcal{O} b_{M}\right) \longrightarrow 0,
$$

where $\bar{\varphi}^{*}(\operatorname{pr})$ is the pull-back of the projection pr : $\left.E\right|_{M} \rightarrow \mathcal{O} b_{M}$.

Let $\left.v \in E\right|_{\varphi(0)}$ be the element so that $s \circ \varphi=v \xi^{n}+O\left(\xi^{n+1}\right)$. Thus $\bar{\varphi}^{*}(d s)=$ $d\left(v \xi^{n}\right)=n v \xi^{n-1} d \xi$.

Let $\bar{\sigma}=\operatorname{pr} \circ \sigma$. Because (4.6) is exact, we have the vanishing $\bar{\varphi}^{*}(\bar{\sigma})\left(\bar{\varphi}^{*}(d s)\right)=0$, which proves that $\bar{\sigma}(v)=0$; hence $\bar{\sigma} \circ s \circ \varphi=O\left(\xi^{n+1}\right)$. Thus the section $s$ satisfies the cone reduction criterion (Lemma 4.4). This proves the corollary.

Assume there is a vector bundle (locally free sheaf of $\mathcal{O}_{\mathcal{M}}$-modules) $F$ that surjects onto $\mathcal{O} b_{\mathcal{M} / \mathcal{S}}$. This homomorphism induces a flat morphism $F \rightarrow h^{1} / h^{0}\left(\left(E^{\bullet}\right)^{\vee}\right)$ (cf. (3.4)), which pulls back $\left[\mathbf{c}_{\mathcal{M}}\right]$ to a cycle $\left[C_{\mathcal{M}}\right] \in Z_{*} F$. We let $\tilde{\sigma}$ be the composition

$$
\tilde{\sigma}:\left.\left.\left.F\right|_{U} \longrightarrow \mathcal{O} b_{\mathcal{M} / \mathcal{S}}\right|_{U} \longrightarrow \mathcal{O} b_{\mathcal{M}}\right|_{U} \longrightarrow \mathcal{O}_{U}
$$

which is surjective. Let $F(\tilde{\sigma}) \subset F$ be $\left.F\right|_{\mathcal{M}(\sigma)} \cup \operatorname{ker}\{\tilde{\sigma}\}$, endowed with the reduced structure.

Corollary 4.6. Let the notation be as stated and let $\sigma$ be a surjective homomorphism $\sigma:\left.\mathcal{O} b_{\mathcal{M}}\right|_{U} \rightarrow \mathcal{O}_{U}$ over an open $U$. Then the cycle $\left[C_{\mathcal{M}}\right] \in Z_{*} F$ lies in $Z_{*} F(\tilde{\sigma})$.

Proof. Because of the way $F(\tilde{\sigma})$ is defined, we only need to show that $\left[C_{\mathcal{M}} \times \mathcal{M} U\right] \in$ $Z_{*}\left(F(\tilde{\sigma}) \times_{\mathcal{M}} U\right)$. By replacing $\mathcal{M}$ with the open substack $U$, we can assume that $\sigma$ is regular and surjective on $\mathcal{M}$.

Since the statement is local, we only need to consider the case where $M / S \rightarrow$ $\mathcal{M} / \mathcal{S}$ is as introduced before (4.1). Since $M$ is affine, we can pick $\left.E^{\bullet}\right|_{M}=\left[E^{-1} \rightarrow\right.$ $\left.E^{0}\right]$ so that $\left.E^{\bullet}\right|_{M} \rightarrow \tau^{\geq-1} L_{M / S}^{\bullet}$ is given by (4.1) and that in addition satisfies $\phi^{0}:\left.E^{0} \rightarrow \Omega_{V / S}\right|_{M}$ is an isomorphism. Because of the exact sequence $\left(E^{0}\right)^{\vee} \rightarrow$ $\left(E^{-1}\right)^{\vee} \rightarrow \mathcal{O} b_{M / S} \rightarrow 0$, possibly by replacing $E^{-1} \rightarrow E^{0}$ with a quasi-isomorphic 
complex we can assume $\left.F\right|_{M} \rightarrow \mathcal{O} b_{M / S}$ lifts to a surjective $\left.F\right|_{M} \rightarrow\left(E^{-1}\right)^{\vee}$, thus defining a homomorphism of complexes

$$
\gamma:[0 \rightarrow F] \rightarrow\left[\left(E^{0}\right)^{\vee} \rightarrow\left(E^{-1}\right)^{\vee}\right] .
$$

Two $\gamma^{\prime}$ 's coming from two liftings $F \rightarrow\left(E^{-1}\right)^{\vee}$ are homotopy equivalent, and hence the induced morphism of bundle stacks

$$
\tilde{\gamma}: F \longrightarrow \mathbf{E}=h^{1} / h^{0}\left(\left(E^{\bullet}\right)^{\vee}\right)
$$

is canonically defined.

Let $\tilde{E}^{-1}$ be a locally free sheaf on $V$ such that $\left.\tilde{E}^{-1}\right|_{M} \cong E^{-1}$. This is possible since $V$ is affine. By the same reason, we can lift $\phi^{-1} \in I_{M} / I_{M}^{2} \otimes_{\mathcal{O}_{M}}\left(E^{-1}\right)^{\vee}$ to an $f \in I_{M} \otimes_{\mathcal{O}_{V}}\left(\tilde{E}^{-1}\right)^{\vee}$. Then since $\phi^{-1}: E^{-1} \rightarrow I_{M} / I_{M}^{2}$ is surjective [2, Thm. 4.5], $M=(f=0) \subset V$. We let $C_{M / V}$ be the normal cone to $M=(f=0)$ in $V$. The cone $C_{M / V}$ canonically embeds in $\left(E^{-1}\right)^{\vee}=\left.\left(\tilde{E}^{-1}\right)^{\vee}\right|_{M}$ via the defining equation $f$.

On the other hand, because the arrow in $\left[I_{M} /\left.I_{M}^{2} \rightarrow \Omega_{V / S}\right|_{M}\right]$ is via sending $u \in$ $I_{M} / I_{M}^{2}$ to its relative differential $\left.d_{/ S} u \in \Omega_{V / S}\right|_{M}$ and because $\phi^{0}$ is an isomorphism, after identifying $E^{0}$ with $\left.\Omega_{V / S}\right|_{M}$ using $\phi^{0}$, the arrow in $\left[E^{-1} \rightarrow E^{0}\right]$ is the relative differential $\left.d_{/ S} f \in \Omega_{V / S}\right|_{M} \otimes_{\mathcal{O}_{M}}\left(\tilde{E}^{-1}\right)^{\vee}$. Thus $\mathcal{O} b_{M / S}=\operatorname{coker}\left\{d_{/ S} f:\left.T_{V / S}\right|_{M} \rightarrow\right.$ $\left.\left(E^{-1}\right)^{\vee}\right\}$. Following the definition of the obstruction sheaf $\mathcal{O} b_{M}$,

$$
\mathcal{O} b_{M}=\operatorname{coker}\left\{d f:\left.T_{V}\right|_{M} \longrightarrow\left(E^{-1}\right)^{\vee}\right\} \text {. }
$$

Finally, we apply Corollary 4.5 to conclude that the support of the cone $C_{M / V}$ lies in the kernel of the composite $\left(E^{-1}\right)^{\vee} \rightarrow \mathcal{O} b_{M} \rightarrow \mathcal{O}_{M}$. Since the pull-back of $\mathbf{c}_{\mathcal{M}}$ to $F$ under (4.8) is the pull-back of $C_{M / V}$ under the surjective $\left.F\right|_{M} \rightarrow\left(E^{-1}\right)^{\vee}$ and since the support of $C_{M / V}$ lies in the kernel of $\left(E^{-1}\right)^{\vee} \rightarrow \mathcal{O}_{M}$, the support of $\left.C_{\mathcal{M}}\right|_{M}$ lies in the kernel $F(\tilde{\sigma})$. This proves the corollary.

Proof of Proposition 4.3. The proof is a direct application of Corollary 4.6. We pick an $M / S \rightarrow \mathcal{M} / \mathcal{S}$ as in the proof of Corollary 4.6. we only need to consider the case where $M \rightarrow \mathcal{M}$ factors through $U \subset \mathcal{M}$. We pick a vector bundle (locally free sheaf) $F$ on $M$ so that $F$ surjects onto $\mathcal{O} b_{M / S}$. This is possible since $M$ is affine. Following the proof of Corollary 4.6 $\gamma: F \rightarrow \mathcal{O} b_{M / S}$ induces a bundle stack homomorphism $\tilde{\gamma}: F \rightarrow \mathbf{E} \times{ }_{\mathcal{M}} M$. Let $\tilde{\sigma}$ be as in (4.7). (Note that $M \rightarrow \mathcal{M}$ factors through $U \subset \mathcal{M}$.) Since for $x \in M$ and $\left(E^{\bullet}\right)^{\vee}=\left[E_{0} \rightarrow E_{1}\right]$ near $x, \operatorname{im}\left(\left.E_{0}\right|_{x}\right) \subset$ $\operatorname{ker}\left(\left.\left.E_{1}\right|_{x} \rightarrow \mathcal{O} b_{M / S}\right|_{x} \stackrel{\sigma}{\longrightarrow} \mathbb{C}\right) \subset \tilde{\gamma}^{-1}\left(\left.\left[E_{1} / E_{0}\right](\sigma)\right|_{x}\right)$, we have $\tilde{\gamma}^{-1}(\mathbf{E}(\sigma))=F(\tilde{\sigma})$. Hence, the statement of the corollary is equivalent to $\tilde{\gamma}^{*}\left[\mathbf{c}_{\mathcal{M}}\right] \in Z^{*} F(\tilde{\sigma})$. But this is what is proved in Corollary 4.6. This proves the proposition.

We have an equivariant version of the corollary. We suppose $\mathcal{M} / \mathcal{S}$ as earlier has a $\mathbb{C}^{*}$-structure; we suppose its relative obstruction theory is $\mathbb{C}^{*}$-equivariant. Let $\mathcal{M}^{c}$ be the $\mathbb{C}^{*}$-fixed part of $\mathcal{M}$. We suppose there is a surjective sheaf homomorphism

$$
\sigma_{c}:\left(\left.\mathcal{O} b_{\mathcal{M}}\right|_{\mathcal{M}^{c}}\right)^{\mathbb{C}^{*}} \longrightarrow \mathcal{O}_{\mathcal{M}^{c}}
$$

from the $\mathbb{C}^{*}$-fixed part to $\mathcal{O}_{\mathcal{M}^{c}}$, and let $F$ be a $\mathbb{C}^{*}$-locally free sheaf of $\mathcal{O}_{\mathcal{M}}$-modules and $F \rightarrow \mathcal{O} b_{\mathcal{M}}$ be a $\mathbb{C}^{*}$-equivariant surjective homomorphism. We let $\sigma$ be the composite

$$
\sigma:\left.\left.\left.F\right|_{\mathcal{M}^{c}} \longrightarrow \mathcal{O} b_{\mathcal{M} / \mathcal{S}}\right|_{\mathcal{M}^{c}} \longrightarrow \mathcal{O} b_{\mathcal{M}}\right|_{\mathcal{M}^{c}} \longrightarrow\left(\left.\mathcal{O} b_{\mathcal{M}}\right|_{\mathcal{M}^{c}}\right)^{\mathbb{C}^{*}} \stackrel{\sigma_{c}}{\longrightarrow} \mathcal{O}_{\mathcal{M}^{c}}
$$

where the second arrow is the projection onto the invariant part. 
Lemma 4.7. Let the notation be as earlier and let $C_{\mathcal{M}} \subset F$ be the cone-cycle that is the pull-back of the intrinsic normal cone $\mathbf{c}_{\mathcal{M}}$ (cf. Corollary 4.6). Then the support of the restriction $\left.\left.C_{\mathcal{M}}\right|_{\mathcal{M}^{c}} \subset F\right|_{\mathcal{M}^{c}}$ lies in the kernel vector bundle $\left.F\right|_{\mathcal{M}^{c}}(\sigma)=\operatorname{ker}\left\{\left.F\right|_{\mathcal{M}^{c}} \rightarrow \mathcal{O}_{\mathcal{M}^{c}}\right\}$.

Proof. We let $M / S \rightarrow \mathcal{M} / \mathcal{S}$ be as in the proof of Corollary 4.6. Without loss of generality, we can assume both $S$ and $M$ are $\mathbb{C}^{*}$-schemes and $M / S \rightarrow \mathcal{M} \mathcal{S}$ is a $\mathbb{C}^{*}$ morphism. (We can avoid this assumption by working with the formal completion of $M$ at a closed $p \in M \times{ }_{\mathcal{M}} \mathcal{M}^{c}$; the remaining arguments go through.) We take a $\mathbb{C}^{*}$-equivariant $S$-embedding $M \rightarrow V$ as earlier.

We repeat the proof of Corollary 4.6 . Since the obstruction theory is $\mathbb{C}^{*}$ equivariant, we can choose a $\mathbb{C}^{*}$-complex $\left.E^{\bullet}\right|_{M}=\left[E^{-1} \rightarrow E^{0}\right]$ so that the obstruction theory $\left.E^{\bullet}\right|_{M} \rightarrow \tau^{\geq-1} L_{M / S}^{\bullet}$ is given by a $\mathbb{C}^{*}$-equivariant (4.1). We extend $E^{-1}$ to a $\mathbb{C}^{*}$-equivariant $\tilde{E}^{-1}$ on $V$. Since (4.1) is $\mathbb{C}^{*}$-equivariant, we can choose a $\mathbb{C}^{*}$-equivariant lift $f \in I_{M} \otimes_{\mathcal{O}_{V}}\left(\tilde{E}^{-1}\right)^{\vee}$ of $\phi^{-1}$.

We let $V^{c}$ (resp. $M^{c}$; resp $E_{c}^{i}$ ) be the $\mathbb{C}^{*}$ fixed part of $V$ (resp. $M$; resp. $E^{i}$ ); let $I_{M^{c}}$ be the ideal sheaf of $M^{c} \subset V^{c}$. Then the $\mathbb{C}^{*}$-fixed part $f^{c}:=(f)^{\mathbb{C}^{*}} \in$ $I_{M^{c}} \otimes \mathcal{O}_{V^{c}}\left(\tilde{E}_{c}^{-1}\right)^{\vee}$ defines $M^{c}=\left(f^{c}=0\right) \subset V^{c}$.

On the other hand, since the $\mathbb{C}^{*}$-invariant part of (4.1) is a perfect obstruction theory of $M^{c}$, the cokernel of $d f^{c}$, which is a quotient of $\left(E_{c}^{-1}\right)^{\vee}$, is the obstruction sheaf $\mathcal{O} b_{M^{c}}$ of $M^{c}$. One checks that it is identical to the invariant part $\left(\left.\mathcal{O} b_{M}\right|_{M^{c}}\right)^{\mathbb{C}^{*}}$ (cf. [11]).

We now look at the normal cone $C_{M^{c} / V^{c}}\left(\right.$ resp. $\left.C_{M / V}\right)$ to $M^{c}$ (resp. $\left.M\right)$ in $V^{c}$ (resp. $V)$; it is a subcone of $\left(E_{c}^{-1}\right)^{\vee}$ (resp. $\left.\left(E^{-1}\right)^{\vee}\right)$. By the previous corollary, the cycle $\left[C_{M^{c} / V^{c}}\right]$ is a cycle in $\operatorname{ker}\left\{\left(E_{c}^{-1}\right)^{\vee} \rightarrow \mathcal{O}_{M^{c}}\right\}$, where the arrow is the composite $\left(E_{c}^{-1}\right)^{\vee} \rightarrow \mathcal{O} b_{M^{c}} \stackrel{\sigma_{c}}{\longrightarrow} \mathcal{O}_{M^{c}}$ (as in the statement of the lemma).

We claim that

$$
\left(C_{M / V}\right)^{\mathbb{C}^{*}}=C_{M^{c} / V^{c}} \subset\left(E_{c}^{-1}\right)^{\vee}
$$

To prove this, we consider the graph $\Gamma$ of $t^{-1} f\left(t \in \mathbb{A}^{1}\right)$, considered as a subscheme in $\left(\mathbb{A}^{1} \backslash 0\right) \times\left(\tilde{E}^{-1}\right)^{\vee}$. By viewing it as a family over $\mathbb{A}^{1} \backslash 0$, we can take its $\mathbb{A}^{1}$ flat closure $\bar{\Gamma} \subset \mathbb{A}^{1} \times\left(\tilde{E}^{-1}\right)^{\vee}$. By the definition of normal cone, the central fiber $\bar{\Gamma}_{0}:=\left.\bar{\Gamma}\right|_{t=0}$ is the cone $C_{M / V}$ canonically embedded in $\left(E^{-1}\right)^{\vee}$. Thus $\bar{\Gamma}_{0}=C_{M / V}$ and $\left(\bar{\Gamma}_{0}\right)^{\mathbb{C}^{*}}=\left(C_{M / V}\right)^{\mathbb{C}^{*}}$.

On the other hand, notice that $\Gamma$ is $\mathbb{C}^{*}$-invariant with $\mathbb{C}^{*}$ acting on $\mathbb{A}^{1}$ trivially; the fixed part $\Gamma^{c}:=\Gamma^{\mathbb{C}^{*}}$ of $\Gamma$ is

$$
\Gamma^{c}=\left\{\text { the graph of } t^{-1} f^{c}\right\} \subset\left(\mathbb{A}^{1} \backslash 0\right) \times\left(\tilde{E}_{c}^{-1}\right)^{\vee} .
$$

We let $\overline{\Gamma^{c}}$ be the flat closure of $\Gamma^{c}$ (over $\mathbb{A}^{1}$ ). Thus, $\left.\left(\overline{\Gamma^{c}}\right)\right|_{t=0}=C_{M^{c} / V^{c}} \subset\left(E_{c}^{-1}\right)^{\vee}$.

Therefore, to conclude the lemma we only need to check that $\left.\left(\overline{\Gamma^{c}}\right)\right|_{t=0}=\left(\bar{\Gamma}_{0}\right)^{c}$. Because $\mathbb{C}^{*}$ is reductive, the flatness of $\bar{\Gamma}$ over $\mathbb{A}^{1}$ implies that the fixed part $(\bar{\Gamma})^{\mathbb{C}^{*}}$ is also flat over $\mathbb{A}^{1}$. Then since $(\bar{\Gamma})^{\mathbb{C}^{*}} \times_{\mathbb{A}^{1}}\left(\mathbb{A}^{1} \backslash 0\right)=\Gamma^{c}$ and since $\overline{\Gamma^{c}}$ is $\mathbb{A}^{1}$-flat, we obtain $\overline{\Gamma^{c}}=(\bar{\Gamma})^{\mathbb{C}^{*}}$. This proves $\left.\overline{\Gamma^{c}}\right|_{t=0}=\left(\bar{\Gamma}_{0}\right)^{\mathbb{C}^{*}}$.

Finally, like in the proof of Corollary 4.6. possibly after replacing $\left[E^{-1} \rightarrow E^{0}\right]$ by a quasi-isomorphic complex, we can assume that $\left.\left.F\right|_{M} \rightarrow \mathcal{O} b_{\mathcal{M}}\right|_{M}$ factors through a $\mathbb{C}^{*}$-equivariant $\left.F\right|_{M} \rightarrow\left(E^{-1}\right)^{\vee} \rightarrow \mathcal{O} b_{M}$. This proves the lemma. 


\section{LOCALIZED VIRTUAL CYCLES}

We state and prove the main theorem of this paper.

Theorem 5.1. Let $\mathcal{M} / \mathcal{S}$ be a Deligne-Mumford stack as earlier endowed with a relative perfect obstruction theory. Suppose there is a surjective homomorphism $\sigma:\left.\mathcal{O} b_{\mathcal{M}}\right|_{U} \rightarrow \mathcal{O}_{U}$ on an open $U \subset \mathcal{M}$. Let $\mathcal{M}(\sigma)=\mathcal{M} \backslash U$ be the degeneracy locus of $\sigma$. Then $\mathcal{M}$ admits a localized virtual cycle

$$
[\mathcal{M}]_{\text {loc }}^{\text {vir }} \in A_{*} \mathcal{M}(\sigma)
$$

It relates to its ordinary virtual cycle by $\iota_{*}[\mathcal{M}]_{\mathrm{loc}}^{\mathrm{vir}}=[\mathcal{M}]^{\mathrm{vir}}$, where $\iota: \mathcal{M}(\sigma) \rightarrow \mathcal{M}$ is the inclusion.

Proof. Let $E^{\bullet} \rightarrow L_{\mathcal{M} / \mathcal{S}}$ be the obstruction theory of $\mathcal{M} / \mathcal{S}$. Let $\mathbf{E}=h^{1} / h^{0}\left(\left(E^{\bullet}\right)^{\vee}\right)$ and let $\left[\mathbf{c}_{\mathcal{M}}\right] \in Z_{*} \mathbf{E}$ be the corresponding intrinsic virtual cycle 2 . The cosection $\sigma$ defines a surjective bundle stack morphism $\tilde{\sigma}:\left.\mathbf{E}\right|_{U} \rightarrow \mathbb{C}_{U}$. As earlier, we let $\mathbf{E}(\sigma)=\left.\mathbf{E}\right|_{\mathcal{M}(\sigma)} \cup \operatorname{ker}\{\tilde{\sigma}\}$, endowed with the reduced structure; $\mathbf{E}(\sigma)$ is a closed substack of $\mathbf{E}$.

By Proposition 4.3, $\left[\mathbf{c}_{\mathcal{M}}\right]$ is a cycle in $Z_{*} \mathbf{E}(\sigma)$. We apply the localized Gysin map constructed in Proposition 3.3 to define

$$
[\mathcal{M}]_{\text {loc }}^{\text {vir }}=s_{\mathbf{E}, \sigma}\left(\left[\mathbf{c}_{\mathcal{M}}\right]\right) \in A_{*} \mathcal{M}(\sigma) .
$$

By the property of the localized Gysin map, we have $\iota_{*}[\mathcal{M}]_{\text {loc }}^{\text {vir }}=[\mathcal{M}]^{\text {vir }}$. This proves the theorem.

Like the ordinary virtual cycle, the localized virtual cycle is expected to remain constant in some naturally arisen situations. We let $\mathcal{M} / \mathcal{S}$ as earlier be a DM stack over a smooth Artin stack $\mathcal{S}, \mathcal{M} \rightarrow \mathcal{S}$ representable, with a relative perfect obstruction theory $E^{\bullet} \rightarrow L_{\mathcal{M} / \mathcal{S}}$. We let $0 \in T$ be a pointed smooth affine curve. We suppose $\mathcal{N} / \mathcal{S}$ is a DM stack over $\mathcal{S}, \mathcal{N} \rightarrow \mathcal{S}$ representable, together with a morphism $\pi: \mathcal{N} \rightarrow T$ such that

$$
\mathcal{M} \cong \mathcal{N} \times{ }_{T} 0 .
$$

We assume there is a perfect relative obstruction theory $F^{\bullet} \rightarrow L_{\mathcal{N} / \mathcal{S}}^{\bullet}$ that is compatible to that of $\mathcal{M} / \mathcal{S}$ in that we have a homomorphism of distinguished triangles in the derived category $D(\mathcal{M})$ :

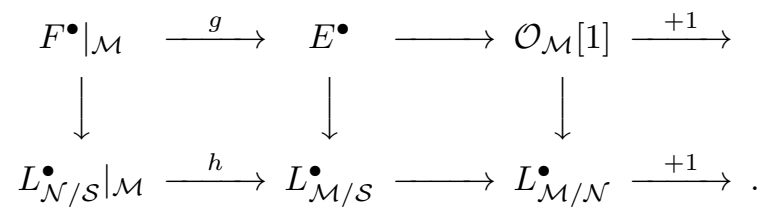

This way, the (relative) obstruction sheaf $\mathcal{O} b_{\mathcal{N} / \mathcal{S}}=h^{1}\left(\left(F^{\bullet}\right)^{\vee}\right)$ fits into the exact sequence $\left.\mathcal{O}_{\mathcal{M}} \rightarrow \mathcal{O} b_{\mathcal{M} / \mathcal{S}} \rightarrow \mathcal{O} b_{\mathcal{N} / \mathcal{S}}\right|_{\mathcal{M}} \rightarrow 0$. Applying the construction of the (absolute) obstruction sheaves, we obtain the exact sequence

$$
\left.\mathcal{O}_{\mathcal{M}} \longrightarrow \mathcal{O} b_{\mathcal{M}} \longrightarrow \mathcal{O} b_{\mathcal{N}}\right|_{\mathcal{M}} \longrightarrow 0 \text {. }
$$

We suppose there is an open $\mathcal{U} \subset \mathcal{N}$ and a surjective homomorphism

$$
\sigma_{\mathcal{U}}:\left.\mathcal{O} b_{\mathcal{N}}\right|_{\mathcal{U}} \longrightarrow \mathcal{O}_{\mathcal{U}}
$$


We let $U=\mathcal{U} \times_{\mathcal{N}} \mathcal{M}$. Let $\sigma:\left.\mathcal{O} b_{\mathcal{M}}\right|_{U} \rightarrow \mathcal{O}_{U}$ be the composition of $\left.\mathcal{O} b_{\mathcal{M}} \rightarrow \mathcal{O} b_{\mathcal{N}}\right|_{\mathcal{M}}$ with $\left.\sigma_{\mathcal{U}}\right|_{U}$. As earlier, we let $\mathcal{N}\left(\sigma_{\mathcal{U}}\right)=\mathcal{N} \backslash \mathcal{U}$ and $\mathcal{M}(\sigma)=\mathcal{M} \backslash U$ be the degeneracy loci of $\sigma_{\mathcal{U}}$ and $\sigma$. Note that $\mathcal{M}(\sigma)=\mathcal{N}\left(\sigma_{\mathcal{U}}\right) \times_{T} 0$.

We let $\tau: 0 \rightarrow T$ be the inclusion and let $\tau^{!}: A_{*} \mathcal{N}\left(\sigma_{\mathcal{U}}\right) \rightarrow A_{*} \mathcal{M}(\sigma)$ be the Gysin map that "intersects" with $0 \in T$.

Theorem 5.2. Let the notation be as stated; let $[\mathcal{N}]_{\text {loc }}^{\mathrm{vir}} \in A_{*} \mathcal{N}\left(\sigma_{\mathcal{U}}\right)$ and $[\mathcal{M}]_{\text {loc }}^{\mathrm{vir}} \in$ $A_{*} \mathcal{M}(\sigma)$ be the localized virtual cycles. Then $[\mathcal{M}]_{\mathrm{loc}}^{\mathrm{vir}}=\tau^{!}\left([\mathcal{N}]_{\mathrm{loc}}^{\mathrm{vir}}\right)$.

We will prove the theorem by applying the rational equivalence inside the deformations of ambient cone-stacks constructed by Kim-Kresch-Pantev [17.

We begin with recalling the convention used in [17. For an object $G^{\bullet}$ in the derived category $D(\mathcal{M})$ of coherent sheaves on $\mathcal{M}$, we denote $p_{\mathcal{M}}^{*} G^{\bullet} \otimes p_{\mathbb{P}^{1}}^{*} \mathcal{O}(-1)$ by $G^{\bullet}(-1)$, where $p_{\mathcal{M}}, p_{\mathbb{P}^{1}}$ are the two projections of $\mathcal{M} \times \mathbb{P}^{1}$. Accordingly, whenever we see a $G^{\bullet} \in D(\mathcal{M})$ appearing in a sequence involving elements in $D\left(\mathcal{M} \times \mathbb{P}^{1}\right)$, we understand it as $p_{\mathcal{M}}^{*} G^{\bullet}$.

For the top line in (5.1), we mimick the construction of [17. Let $[x, y]$ be the homogeneous coordinates of $\mathbb{P}^{1}$, and let $\tilde{g}:\left.\left.F^{\bullet}\right|_{\mathcal{M}}(-1) \rightarrow F^{\bullet}\right|_{\mathcal{M}} \oplus E^{\bullet}$ be defined by $(x \cdot 1, y \cdot g)$. The mapping cone $c(\tilde{g})$ of $\tilde{g}$ is locally quasi-isomorphic to a two-term complex of locally free sheaves and fits into the distinguished triangle

$$
\left.\left.F^{\bullet}\right|_{\mathcal{M}}(-1) \stackrel{\tilde{g}}{\longrightarrow} F^{\bullet}\right|_{\mathcal{M}} \oplus E^{\bullet} \longrightarrow c(\tilde{g}) \stackrel{+1}{\longrightarrow} .
$$

Following [17, $h^{1} / h^{0}\left(c(\tilde{g})^{\vee}\right)$ is a vector bundle stack over $\mathcal{M} \times \mathbb{P}^{1}$, thus flat over $\mathbb{P}^{1}$; its fibers over $a=[1,0]$ and $b=[0,1] \in \mathbb{P}^{1}$ are

$$
\begin{gathered}
h^{1} / h^{0}\left(c(\tilde{g})^{\vee}\right) \times_{\mathbb{P}^{1}} a=h^{1} / h^{0}\left(\left(E^{\bullet}\right)^{\vee}\right), \\
h^{1} / h^{0}\left(c(\tilde{g})^{\vee}\right) \times_{\mathbb{P}^{1}} b=h^{1} / h^{0}\left(\left(\left.F^{\bullet}\right|_{\mathcal{M}}\right)^{\vee}\right) \times \mathbb{A}^{1} .
\end{gathered}
$$

Here the $\mathbb{A}^{1}$ in the product is the fiber of the vector bundle $h^{1} / h^{0}\left(\left[0 \rightarrow \mathcal{O}_{\mathcal{M}}\right]\right) \cong \mathbb{C}_{\mathcal{M}}$.

We let $\mathbf{M}_{\mathcal{N} / \mathcal{S}}^{0}$ be the deformation of $\mathcal{N}$ to its normal cone $\mathbf{c}_{\mathcal{N} / \mathcal{S}}$; let $\mathbf{c}_{\mathcal{M} \times \mathbb{P}^{1} / \mathbf{M}_{\mathcal{N} / \mathcal{S}}^{0}}$ be the normal cone to $\mathcal{M} \times \mathbb{P}^{1}$ in $\mathbf{M}_{\mathcal{N} / \mathcal{S}}^{0}$. We let $\mathbf{e}=h^{1} / h^{0}\left(c(\tilde{g})^{\vee}\right)$, which is a bundle stack over $\mathcal{M} \times \mathbb{P}^{1}$. According to [17, we have canonical closed immersions

$$
\mathbf{c}_{\mathcal{N} / \mathcal{S}} \subset h^{1} / h^{0}\left(\left(F^{\bullet}\right)^{\vee}\right) \text { and } \mathcal{D}:=\mathbf{c}_{\mathcal{M} \times \mathbb{P}^{1} / \mathbf{M}_{\mathcal{N} / \mathcal{S}}^{0}} \subset \mathbf{e}=h^{1} / h^{0}\left(c(\tilde{g})^{\vee}\right) .
$$

We see that $\mathcal{D}$ is flat over $\mathbb{P}^{1}-b$; for $\mathbf{E}:=h^{1} / h^{0}\left(\left(E^{\bullet}\right)^{\vee}\right), \mathbf{e} \times_{\mathbb{P}^{1}} a \cong \mathbf{E}$ and

$$
a^{!}[\mathcal{D}]=\left[\mathcal{D} \times_{\mathbb{P}^{1}} a\right]=\left[\mathbf{c}_{\mathcal{M} / \mathcal{S}}\right] \in Z_{*} \mathbf{E} .
$$

(Following [10, for $c \in \mathbb{P}^{1}$ we define $c^{!}[\mathcal{D}]=\left[\mathcal{D}^{\mathrm{fl}} \times_{\mathbb{A}^{1}} c\right]$, where $\mathcal{D}^{\mathrm{fl}}$ is the $\mathbb{P}^{1}$-flat part of $\mathcal{D}$.) Consequently, (the flat part of) $\mathcal{D}$ provides a rational equivalence between $\left[\mathbf{c}_{\mathcal{M} / \mathcal{S}}\right]$ and $b^{!}[\mathcal{D}]$.

We let $\mathcal{D}_{b}=\mathcal{D} \times_{\mathbb{P}^{1}} b$. In [30] and [17, a canonical rational equivalence $[\mathcal{R}] \in$ $W_{*}\left(\mathcal{D}_{b}\right)$ is constructed such that

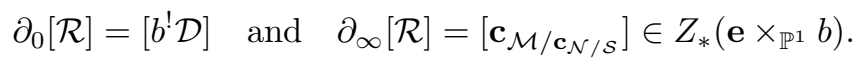

Combining, we obtain a pair of rational equivalences giving the equivalence of

$$
\left[\mathbf{c}_{\mathcal{M} / \mathcal{S}}\right] \sim\left[\mathbf{c}_{\left.\mathcal{M} / \mathbf{c}_{\mathcal{N} / \mathcal{S}}\right]} \text { via }([\mathcal{D}],[\mathcal{R}]) .\right.
$$

This rational equivalence implies $\tau^{!}[\mathcal{N}]^{\mathrm{vir}}=[\mathcal{M}]^{\mathrm{vir}}$.

To prove the constancy of the localized virtual cycles, we need to show that both $[\mathcal{D}]$ and $[\mathcal{R}]$ lie in the appropriate kernel bundle stack. First, $\sigma_{\mathcal{U}}:\left.\mathcal{O}_{\mathcal{N}}\right|_{\mathcal{U}} \rightarrow \mathcal{O}_{\mathcal{U}}$ 
induces $\left.\left(F^{\bullet}\right)^{\vee}\right|_{\mathcal{U}} \rightarrow \mathcal{O}_{\mathcal{U}}[-1]$, which, together with the $\left.\left(E^{\bullet}\right)^{\vee}\right|_{U} \rightarrow \mathcal{O}_{U}[-1]$ induced by $\sigma$, defines the two right vertical arrows $\beta_{1}$ and $\beta_{2}$ shown below, making the right square a commutative square of homomorphisms of complexes. Using $\beta_{1}$ and $\beta_{2}$, we construct a homomorphism of complexes $\sigma^{\prime}$ (as shown below), which, together with the $\beta_{i}$, defines an arrow between distinguished triangles (after restricting to $\left.U \times \mathbb{P}^{1}\right)$ :

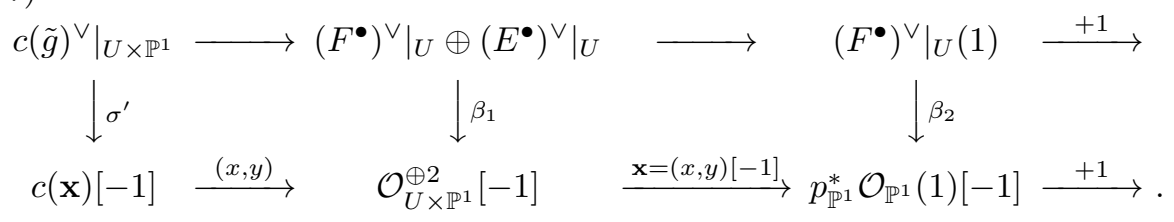

Let $L$ be the line bundle $\mathcal{O}_{\mathbb{P}^{1}}(-1)$. Then, $c(\mathbf{x}) \cong_{q i s} p_{\mathbb{P}^{1}}^{*} L$; thus

$$
h^{1} / h^{0}(c(\mathbf{x})[-1]) \cong p_{\mathbb{P}^{1}}^{*} L,
$$

which is a line bundle on $\mathcal{M} \times \mathbb{P}^{1}$. On the other hand, since both $\beta_{1}$ and $\beta_{2}$ are defined and surjective on $U \times \mathbb{P}^{1}, \bar{\sigma}$ is surjective on $U \times \mathbb{P}^{1}$. Thus $\bar{\sigma}$ induces a surjective bundle-stack homomorphism (which we still denote by $\bar{\sigma}$ )

$$
\bar{\sigma}:\left.\left.\mathbf{e}\right|_{U \times \mathbb{P}^{1}} \longrightarrow p_{\mathbb{P}^{1}}^{*} L\right|_{U \times \mathbb{P}^{1}} .
$$

We let $\mathbf{e}(\bar{\sigma})$ be the union of $\left.\mathbf{e}\right|_{\mathcal{M}(\sigma) \times \mathbb{P}^{1}}$ with the kernel $\operatorname{ker}\{\bar{\sigma}\}$, endowed with the reduced structure; $\mathbf{e}(\bar{\sigma}) \subset \mathbf{e}$ is closed. As mentioned already, we let $W_{*} \mathbf{e}_{b}$ be the group of rational equivalences of $\mathbf{e}_{b}=\mathbf{e} \times_{\mathbb{P}^{1}} b$.

Lemma 5.3. The cycle $[\mathcal{D}] \in Z_{*} \mathbf{e}$ and the rational equivalence $[\mathcal{R}] \in W_{*} \mathbf{e}_{b}$ lie in $Z_{*} \mathbf{e}(\bar{\sigma})$ and $W_{*} \mathbf{e}(\bar{\sigma})_{b}$, respectively.

Proof. Since $\mathcal{R} \subset \mathcal{D} \times \times_{\mathbb{P}^{1}} b$, the support of $\mathcal{R}$ lies in $\mathcal{D}$. Thus we only need to show that the support of $\mathcal{D}$ lies in $\mathbf{e}(\bar{\sigma})$. Furthermore, since $\left.\mathbf{e}\right|_{\mathcal{M}(\sigma) \times \mathbb{P}^{1}}=\left.\mathbf{e}(\bar{\sigma})\right|_{\mathcal{M}(\sigma) \times \mathbb{P}^{1}}$, to prove the lemma we only need to show that the support of $\mathcal{D} \times{ }_{\mathcal{M} \times \mathbb{P}^{1}}\left(U \times \mathbb{P}^{1}\right)$ lies in $\left.\mathbf{e}(\bar{\sigma})\right|_{U \times \mathbb{P}^{1}}$. Thus, it suffices to prove the lemma in case $\mathcal{N}=\mathcal{U}$; namely, $\sigma_{\mathcal{U}}$ is regular and surjective everywhere.

In the following, we assume $\sigma_{\mathcal{N}}: \mathcal{O} b_{\mathcal{N}} \rightarrow \mathcal{O}_{\mathcal{N}}$ is regular and surjective on $\mathcal{N}$. Since the statement of the lemma is local, it suffices to investigate the situation over $N / S \rightarrow \mathcal{N} / \mathcal{S}$ for $S$ smooth over $\mathcal{S}, N \rightarrow \mathcal{N}$ étale, and $N \subset \mathcal{N} \times \mathcal{S} S$ an affine open subscheme.

We then pick a smooth affine scheme $V$ over $S$ and $T$, an embedding $N \rightarrow V$ that is both an $S$ - and $T$-embedding. Using $N \rightarrow V$, we have a representative

$$
\tau^{\geq-1} L_{N / S}^{\bullet}=\left[I_{N} /\left.I_{N}^{2} \rightarrow \Omega_{V / S}\right|_{N}\right] \quad \text { and } \quad \tau^{\geq-1} L_{M / S}^{\bullet}=\left[I_{M} /\left.I_{M}^{2} \rightarrow \Omega_{V / S}\right|_{M}\right]
$$

where $M=N \times_{T} 0$ is the corresponding chart of $\mathcal{M}, I_{N}$ and $I_{M}$ are the ideal sheaves of $N \subset V$ and $M \subset V$, respectively.

Since $N$ and $V$ are affine, we can assume that there is a vector bundle (locally free sheaf) $F$ on $N$ (resp. $E$ on $M$ ) so that

$$
\left.F^{\bullet}\right|_{N}=\left[\left.F^{\vee} \rightarrow \Omega_{V / S}\right|_{N}\right] \text { and }\left.\quad E^{\bullet}\right|_{M}=\left[\left.E^{\vee} \rightarrow \Omega_{V / S}\right|_{M}\right],
$$


and the diagram (5.1) restricted to $M$ is represented by the following commuting homomorphisms of complexes of sheaves:

$$
\begin{aligned}
& {\left.\left[\left.F^{\vee} \rightarrow \Omega_{V / S}\right|_{N}\right]\right|_{M} \stackrel{g_{N}}{\longrightarrow}\left[\left.E^{\vee} \rightarrow \Omega_{V / S}\right|_{M}\right] \longrightarrow \quad\left[\mathcal{O}_{M} \rightarrow 0\right]} \\
& \left.\downarrow\left[\phi^{-1}, \phi^{0}\right]\right|_{M} \quad \downarrow\left[\psi^{-1}, \psi^{0}\right] \downarrow \\
& \left.\tau^{\geq-1} L_{N / S}^{\bullet}\right|_{M} \longrightarrow \tau^{\geq-1} L_{M / S}^{\bullet} \longrightarrow\left[I_{M \subset N} / I_{M \subset N}^{2} \rightarrow 0\right]
\end{aligned}
$$

which in addition satisfy $\phi^{0}=\mathrm{id}:\left.\left.\Omega_{V / S}\right|_{N} \rightarrow \Omega_{V / S}\right|_{N}$ and $\psi^{0}=\mathrm{id}$, and the part of the top line at place $[-1]$ is an exact sequence

$$
\left.0 \longrightarrow F^{\vee}\right|_{M} \longrightarrow E^{\vee} \longrightarrow \mathcal{O}_{M} \longrightarrow 0 .
$$

Here, for the terms in the second line, we use representatives (5.9).

Since $V$ is affine, we can split this exact sequence to get $\left.E^{\vee} \cong F^{\vee}\right|_{M} \oplus \mathcal{O}_{M}$. We then extend $F$ to a vector bundle (locally free sheaf) $\tilde{F}$ on $V$; because of the isomorphism $\left.E^{\vee} \cong F^{\vee}\right|_{M} \oplus \mathcal{O}_{M}, \tilde{F} \oplus \mathcal{O}_{V}$ is an extension of $E$.

We now give an explicit description of $\left.\mathbf{e}\right|_{M \times \mathbb{P}^{1}}\left(:=\mathbf{e} \times_{\mathcal{M} \times \mathbb{P}^{1}}\left(M \times \mathbb{P}^{1}\right)\right)$. By the canonical construction of $c(\tilde{g})$, we have a canonical isomorphism

$$
\left.\mathbf{e}\right|_{M \times \mathbb{P}^{1}} \cong h^{1} / h^{0}\left(c\left(\tilde{g}_{N}\right)^{\vee}\right),
$$

where $\tilde{g}_{N}=\left(x \cdot 1, y \cdot g_{N}\right)$ is

$$
\tilde{g}_{N}:\left.\left.\left[\left.F^{\vee} \rightarrow \Omega_{V / S}\right|_{N}\right]\right|_{M}(-1) \longrightarrow\left[\left.F^{\vee} \rightarrow \Omega_{V / S}\right|_{N}\right]\right|_{M} \oplus\left[\left.E^{\vee} \rightarrow \Omega_{V / S}\right|_{M}\right]
$$

(cf. $\tilde{g}$ in (5.3) ). Using the isomorphism $\left.E \cong F\right|_{M} \oplus \mathcal{O}_{M}$ and the fact that the arrow $\left.F^{\vee}\right|_{M} \rightarrow E^{\vee}$ is the inclusion under the splitting, we see that the mapping cone

$$
c\left(\tilde{g}_{N}\right) \cong_{q i s}\left[\left.\left.F^{\vee}\right|_{M} \rightarrow \Omega_{V / S}\right|_{M}\right](1) \oplus\left[\mathcal{O}_{M} \rightarrow 0\right] .
$$

Thus, following the convention that $\left.F\right|_{M}(-1)=\left.p_{M}^{*} F\right|_{M} \otimes p_{\mathbb{P}^{1}}^{*} L$ is a vector bundle on $M \times \mathbb{P}^{1}$, where $L \cong \mathcal{O}_{\mathbb{P}^{1}}(-1)$, we obtain a tautological flat bundle stack morphism

$$
\Phi: F_{M}(-1) \times \mathbb{A}^{1} \longrightarrow \mathbf{e}_{N}=h^{1} / h^{0}\left(c\left(\tilde{g}_{N}\right)^{\vee}\right) .
$$

We let $\left.\left.\mathcal{D}\right|_{M \times \mathbb{P}^{1}} \subset \mathbf{e}\right|_{M \times \mathbb{P}^{1}}$ be the pull-back of $\mathcal{D} \subset \mathbf{e}$ using the isomorphism (5.12) and let

$$
D=\left.\Phi^{*}\left(\left.\mathcal{D}\right|_{M \times \mathbb{P}^{1}}\right) \subset F\right|_{M}(-1) \times \mathbb{A}^{1} .
$$

We next describe the pull-back $\Phi^{*}(\mathbf{e}(\bar{\sigma}))$. ( $\bar{\sigma}$ is defined in (5.8).) Like the construction of $\sigma^{\prime}$ in (5.7), the surjective homomorphism $\sigma_{\mathcal{N}}: \mathcal{O} b_{\mathcal{N}} \rightarrow \mathcal{O}_{\mathcal{N}}$ (surjectivity is assumed at the beginning of the proof) induces a surjective homomorphism

$$
\delta: F \longrightarrow h^{1}\left(\left(F^{\bullet}\right)^{\vee}\right) \longrightarrow \overline{\mathbb{C}}_{N} .
$$

Cf. (5.10). Pulling back $\delta$ to $N \times \mathbb{P}^{1}$ and twisting it by $\mathcal{O}_{\mathbb{P}^{1}}(-1)$, we obtain the second arrow shown below, which composed with the projection to the first factor of $F(-1) \oplus \mathbb{C}_{N \times \mathbb{P}^{1}}$ defines $\gamma_{N}$ :

$$
\gamma_{N}: F(-1) \oplus \mathbb{C}_{N \times \mathbb{P}^{1}} \stackrel{\mathrm{pr}}{\longrightarrow} F(-1) \longrightarrow \bar{p}_{\mathbb{P}^{1}}^{*} L,
$$

where $\bar{p}_{\mathbb{P}^{1}}: N \times \mathbb{P}^{1} \rightarrow \mathbb{P}^{1}$ is the projection.

We let $K$ be the kernel bundle of $\gamma_{N}$. It is direct to check that

$$
\left.K\right|_{M \times \mathbb{P}^{1}}=\left.\Phi^{*}(\mathbf{e}(\bar{\sigma})) \subset F\right|_{M}(-1) \times \mathbb{A}^{1} .
$$

\footnotetext{
${ }^{2}$ The bottom line of (5.11) is a truncated version of the distinguished triangle of cotangent complexes for $M \rightarrow N \rightarrow S$. It is not necessarily exact
} 
This way, $[\mathcal{D}] \in Z_{*} \mathbf{e}(\bar{\sigma})$ is equivalent to the reduced part $D_{\text {red }}$ (of $D$ ) lying in $\left.K\right|_{M \times \mathbb{P}^{1}}$.

To prove $\left.D_{\text {red }} \subset K\right|_{M \times \mathbb{P}^{1}}$, we give a graph construction of $D$. We pick a lifting $f_{1} \in I_{N} \otimes_{\mathcal{O}_{V}} \tilde{F}$ of $\phi^{-1} \in I_{N} / I_{N}^{2} \otimes_{\mathcal{O}_{N}} F$ and extend $f_{1}$ to a lifting $\left(f_{1}, f_{2}\right) \in$ $I_{M} \otimes_{\mathcal{O}_{V}}\left(\tilde{F} \oplus \mathbb{C}_{V}\right)$ of $\psi^{-1}$. After shrinking $V$ if necessary, $f_{1}=0$ defines $N$ and $\left(f_{1}, f_{2}\right)=0$ defines $M$. By shrinking $T$ if necessary, we can pick a uniformizing parameter $t \in \Gamma\left(\mathcal{O}_{T}\right)$ so that $t=0$ consists of a single point $0 \in T$.

We next view $x^{-1}$ as a meromorphic section of $L=\mathcal{O}_{\mathbb{P}^{1}}(-1)$ with no zero and with its only pole at $b=[0,1]$. Then $\left((t x)^{-1} f_{1}, t^{-1} f_{2}\right)$ is a section of $F(-1) \oplus \mathbb{C}_{N \times \mathbb{P}^{1}}$ over $(N \backslash M) \times\left(\mathbb{P}^{1}-b\right)$; we denote by

$$
\left.\Gamma \subset\left(F(-1) \times \mathbb{A}^{1}\right)\right|_{(N \backslash M) \times\left(\mathbb{P}^{1}-b\right)}
$$

this section (graph). We let $\bar{\Gamma}$ be the closure of $\Gamma$ in $F(-1) \times \mathbb{A}^{1}$. According to the construction in [17,

$$
D=\bar{\Gamma} \times\left._{N \times \mathbb{P}^{1}}\left(M \times \mathbb{P}^{1}\right) \subset F\right|_{M}(-1) \times \mathbb{A}^{1} .
$$

We now prove $\left.D_{\text {red }} \subset K\right|_{M \times \mathbb{P}^{1}}$. First, let

$$
\left.\Gamma_{1} \subset\left(F \oplus \mathbb{C}_{N}\right)\right|_{N \backslash M}
$$

be the graph of $\left(t^{-1} f_{1}, t^{-1} f_{2}\right)$ (recall that $t \in \Gamma\left(\mathcal{O}_{T}\right)$ and $M=N \cap\left(f_{2}=0\right)$ ); let $\overline{\Gamma_{1}}$ be its closure in $F \oplus \mathbb{C}_{N}$; then $\overline{\Gamma_{1}} \times{ }_{N} M$ is the normal cone $C_{M / V}$ embedded in $\left.F\right|_{M} \oplus \mathbb{C}_{M}=\left.F\right|_{M} \times \mathbb{A}^{1}$ by the defining equation $\left(f_{1}, f_{2}\right)$ of $M$.

We next form the commutative diagram

$$
\begin{array}{ccc}
\left.\left(F(-1) \oplus \mathbb{C}_{N \times \mathbb{P}^{1}}\right)\right|_{N \times\left(\mathbb{P}^{1}-b\right)} & \left.\stackrel{(x \cdot 1,1)}{\longrightarrow} \bar{p}_{N}^{*}\left(F \oplus \mathbb{C}_{N}\right)\right|_{N \times\left(\mathbb{P}^{1}-b\right)} \\
\downarrow^{\gamma_{N}} & & \downarrow_{\left(\bar{p}_{N}^{*} \delta, 0\right)} \\
\left.\bar{p}_{\mathbb{P}^{1}}^{*} L\right|_{N \times\left(\mathbb{P}^{1}-b\right)} & \stackrel{x}{\longrightarrow} & \left.\mathbb{C}_{N \times \mathbb{P}^{1}}\right|_{N \times\left(\mathbb{P}^{1}-b\right)} .
\end{array}
$$

Note that the two horizontal arrows are isomorphisms of vector bundles. By our construction, $\Gamma$ is the preimage of $\bar{p}_{N}^{*} \Gamma_{1}$ under the top horizontal arrow. Therefore, $\left.\bar{\Gamma}\right|_{N \times\left(\mathbb{P}^{1}-b\right)}$ is the preimage of $\left.\bar{p}_{N}^{*} \bar{\Gamma}_{1}\right|_{N \times\left(\mathbb{P}^{1}-b\right)}$, and thus

$$
\left.\left.D\right|_{M \times\left(\mathbb{P}^{1}-b\right)} \subset\left(\left.p_{M}^{*} F\right|_{M} \times \mathbb{A}^{1}\right)\right|_{M \times\left(\mathbb{P}^{1}-b\right)}
$$

is the preimage of $p_{M}^{-1} C_{M / V} \subset p_{M}^{*}\left(\left.F\right|_{M} \oplus \mathbb{C}_{M}\right)$. This proves that $\mathcal{D}$ is flat over $\mathbb{P}^{1}-b$ and also the identity (5.5).

Furthermore, by Corollary 4.5, the support of $\left.\left.C_{M / V} \subset E\right|_{M} \cong F\right|_{M} \oplus \mathbb{C}_{M}$ lies in the kernel of $(\delta, 0):\left.F\right|_{M} \oplus \mathbb{C}_{M} \rightarrow \mathbb{C}_{M}$. Thus the support of $p_{M}^{-1} C_{M / V}$ lies in the kernel of $\left(\bar{p}_{N}^{*} \delta, 0\right)$, and the support of $\left.D\right|_{M \times\left(\mathbb{P}^{1}-b\right)}$ lies in the kernel of $\gamma_{N}$.

It remains to show that every irreducible component of $D$ that lies over $M \times_{\mathbb{P}^{1}} b$ lies in $\left.K\right|_{M \times \mathbb{P}^{1}}$ (cf. (5.16)). Let $A \subset D$ be an irreducible component lying over $M \times_{\mathbb{P}^{1}} b$, and let $v \in A$ be a general closed point. Since $D \subset \bar{\Gamma}-\Gamma$, we can find a smooth curve $0 \in S$ and a morphism $\tilde{\rho}:(0, S) \rightarrow(v, \bar{\Gamma})$ so that $\tilde{\rho}(S \backslash 0) \in \Gamma$. We let $\left(\rho_{1}, \rho_{2}\right): S \rightarrow M \times \mathbb{P}^{1}$ be $\tilde{\rho}$ composed with the projection $\bar{\Gamma} \rightarrow M \times \mathbb{P}^{1}$. By shrinking $S$ if necessary, we can assume $\rho_{2}(S) \subset \mathbb{P}^{1}-a$.

We let $x_{S}:=x \circ \rho_{2}\left(\right.$ resp. $\left.t_{S}:=t \circ \rho_{1}\right)$ be the composition of $x \in \Gamma\left(\mathcal{O}_{\mathbb{P}^{1}-a}\right)$ with $\rho_{2}$ (resp. $t \in \Gamma\left(\mathcal{O}_{T}\right)$ with the composite $S \stackrel{\rho_{1}}{\longrightarrow} M \rightarrow T$ ). Since $v=\lim _{s \rightarrow 0} \tilde{\rho}(s)$, by the definition of $\Gamma$, we have

$$
v=\left(v_{1}, v_{2}\right)=\left(\lim _{s \rightarrow 0}\left(x_{S} \cdot t_{S}\right)^{-1} \cdot f_{1} \circ \rho, \lim _{s \rightarrow 0}\left(t_{S}\right)^{-1} \cdot f_{2} \circ \rho\right) .
$$


Let $u=\rho_{1}(0) \in M$. Repeating the proofs of Lemma 4.4 and Corollary 4.5, the surjective homomorphism $\sigma_{\mathcal{N}}: \mathcal{O}_{\mathcal{N}} \rightarrow \mathcal{O}_{\mathcal{N}}$ forces $v_{1} \in \operatorname{ker}\left\{\delta(u):\left.F\right|_{u} \rightarrow \mathbb{C}\right\}$ (cf. (5.14)). Finally, by the explicit form of $\gamma_{N}$ in (5.15), $\operatorname{ker}\left\{\delta(u):\left.F\right|_{u} \rightarrow \mathbb{C}\right\} \times \mathbb{A}^{1}=$ $\left.K\right|_{u \times b}$. Therefore, $v_{1}$ lies in this kernel, which proves $\left.A \subset K\right|_{M \times \mathbb{P}^{1}}$. This proves the lemma.

Proof of Theorem 5.2. This follows from the fact that the localized Gysin map $s_{\mathbf{e}, \bar{\sigma}}$ preserves rational equivalence.

\section{Application: Localized GW-invariants}

We let $X$ be a smooth quasi-projective variety equipped with a holomorphic two-form $\theta \in \Gamma\left(\Omega_{X}^{2}\right)$. This form induces a cosection of the obstruction sheaf of $\overline{\mathcal{M}}_{g, n}(X, \beta)$, the moduli space of $n$-pointed genus $g$ stable morphisms to $X$ of class $\beta$. This cosection defines a localized virtual class of $\overline{\mathcal{M}}_{g, n}(X, \beta)$, thus localized GW-invariants of $(X, \theta)$.

We begin with the construction of the cosection of the obstruction sheaf of $\overline{\mathcal{M}}_{g, n}(X, \beta)$. For simplicity, we will fix the data $g, n, X$, and $\beta$ for the moment and abbreviate $\overline{\mathcal{M}}_{g, n}(X, \beta)$ to $\mathcal{M}$. We let $f: \mathcal{C} \rightarrow X$ and $\pi: \mathcal{C} \rightarrow \mathcal{M}$ be the universal family of $\mathcal{M}$. If we denote by $\mathcal{S}$ the Artin stack of genus $g$ connected nodal curves with $n$ marked points, $\mathcal{M}$ is a representable DM stack over $\mathcal{S}$; and the relative obstruction sheaf of the standard relative obstruction theory of $\mathcal{M} / \mathcal{S}$ (cf. [2]) is $\mathcal{O} b_{\mathcal{M} / \mathcal{S}}=R^{1} \pi_{*} f^{*} T_{X}$.

We now show that a holomorphic two-form $\theta$ defines a cosection of $\mathcal{O} b_{\mathcal{M} / \mathcal{S}}$. Indeed, by viewing it as an anti-symmetric homomorphism

$$
\hat{\theta}: T_{X} \longrightarrow \Omega_{X}, \quad(\hat{\theta}(v), v)=0,
$$

it defines the first arrow in the following sequence of homomorphisms:

$$
R^{1} \pi_{*} f^{*} T_{X} \longrightarrow R^{1} \pi_{*} f^{*} \Omega_{X} \longrightarrow R^{1} \pi_{*} \Omega_{\mathcal{C} / \mathcal{M}} \longrightarrow R^{1} \pi_{*} \omega_{\mathcal{C} / \mathcal{M}}
$$

where the second is induced by $f^{*} \Omega_{X} \rightarrow \Omega_{\mathcal{C} / \mathcal{M}}$, and the last by the tautological $\Omega_{\mathcal{C} / \mathcal{M}} \rightarrow \omega_{\mathcal{C} / \mathcal{M}}$. Because $R^{1} \pi_{*} \omega_{\mathcal{C} / \mathcal{M}} \cong \mathcal{O}_{\mathcal{M}}$, the composite of this sequence provides

$$
\sigma_{\theta}^{\mathrm{rel}}: R^{1} \pi_{*} f^{*} T_{X}=\mathcal{O} b_{\mathcal{M} / \mathcal{S}} \longrightarrow \mathcal{O}_{\mathcal{M}}
$$

The obstruction sheaf of $\mathcal{M}$ is the cokernel of $p^{*} T_{\mathcal{S}} \rightarrow \mathcal{O} b_{\mathcal{M} / \mathcal{S}}$, where $p: \mathcal{M} \rightarrow \mathcal{S}$ is the projection. Using the universal family $f$ and the fact that $R^{1} \pi_{*} f^{*} T_{X}=$ $\mathcal{E} x t_{\pi}^{1}\left(f^{*} \Omega_{X}, \mathcal{O}_{\mathcal{C}}\right)$, we have the exact sequence

$$
\mathcal{E} x t_{\pi}^{1}\left(\Omega_{\mathcal{C} / \mathcal{M}}(x), \mathcal{O}_{\mathcal{C}}\right) \longrightarrow \mathcal{E} x t_{\pi}^{1}\left(f^{*} \Omega_{X}, \mathcal{O}_{\mathcal{C}}\right) \longrightarrow \mathcal{O} b_{\mathcal{M}} \longrightarrow 0,
$$

where the first arrow is induced by $f^{*} \Omega_{X} \rightarrow \Omega_{\mathcal{C} / \mathcal{M}}$. Here $x=x_{1}+\cdots+x_{n}$ denotes the divisor of marked points.

Lemma 6.1. The composition

$$
\mathcal{E} x t_{\pi}^{1}\left(\Omega_{\mathcal{C} / \mathcal{M}}(x), \mathcal{O}_{\mathcal{C}}\right) \longrightarrow \mathcal{E} x t_{\pi}^{1}\left(f^{*} \Omega_{X}, \mathcal{O}_{\mathcal{C}} \stackrel{\sigma_{\theta}^{\mathrm{rel}}}{\longrightarrow} \mathcal{O}_{\mathcal{M}}\right.
$$

is trivial.

Proof. Applying the definition of $\sigma_{\theta}^{\text {rel }}$, one sees that the stated composition is the composition of the sequence

$$
\mathcal{E} x t_{\pi}^{1}\left(\Omega_{\mathcal{C} / \mathcal{M}}(x), \mathcal{O}_{\mathcal{C}}\right) \longrightarrow \mathcal{E} x t_{\pi}^{1}\left(f^{*} \Omega_{X}, \mathcal{O}_{\mathcal{C}}\right) \longrightarrow \mathcal{E} x t_{\pi}^{1}\left(f^{*} T_{X}, \mathcal{O}_{\mathcal{C}}\right) \longrightarrow \mathcal{E} x t_{\pi}^{1}\left(\omega_{\mathcal{C} / \mathcal{M}}^{\vee}, \mathcal{O}_{\mathcal{C}}\right)
$$


that is induced by the sequence

$$
\Theta: \omega_{\mathcal{C} / \mathcal{M}}^{\vee} \longrightarrow f^{*} T_{X} \stackrel{f^{*} \hat{\theta}}{\longrightarrow} f^{*} \Omega_{X} \longrightarrow \Omega_{\mathcal{C} / \mathcal{M}}(x),
$$

where the first arrow is the dual of the composite $f^{*} \Omega_{X} \rightarrow \Omega_{\mathcal{C} / \mathcal{M}} \rightarrow \omega_{\mathcal{C} / \mathcal{M}}$.

We now prove that the composite $\Theta=0$. First, let $\mathcal{C}_{\text {reg }}$ be the smooth loci of the fibers of $\mathcal{C} / \mathcal{M}$. Since $\left.\Omega_{\mathcal{C} / \mathcal{M}}\right|_{\mathcal{C}_{\text {reg }}}=\left.\omega_{\mathcal{C} / \mathcal{M}}\right|_{\mathcal{C}_{\text {reg }}}$ and since $\hat{\theta}$ (in (6.1) ) is anti-symmetric, $\left.\Theta\right|_{\mathcal{C}_{\text {reg }}}$ is anti-symmetric. Therefore, because $\omega_{\mathcal{C} / \mathcal{M}}$ is invertible, $\left.\Theta\right|_{\mathcal{C}_{\text {reg }}}=0$.

Now let $q \in \mathcal{C} \backslash \mathcal{C}_{\text {reg }}$; let $\xi=\pi(q) \in \mathcal{M}$. We pick an affine scheme $M$ and an étale morphism $M \rightarrow \mathcal{M}$ whose image contains $\xi$; let $\bar{\xi} \in M$ be a lift of $\xi$. We let $C$ be an affine open $C \subset \mathcal{C} \times{ }_{\mathcal{M}} M$ such that $(q, \xi) \in \mathcal{C} \times{ }_{\mathcal{M}} M$ lifts to a $\bar{q} \in C$. We let $g: C \rightarrow X$ be the restriction of $f$ to $C$ and let $\bar{\xi} \in M$ be the image of $\bar{q}$ under $C \rightarrow M$. Since both $C$ and $M$ are affine, we can find a closed immersion $M \subset \tilde{M}$ into a smooth scheme $\tilde{M}$ and extend $C / M$ to a family of nodal curves $\tilde{C} / \tilde{M}$ so that the node $\bar{q} \in C_{\bar{\xi}}$ is smoothed in the family $\tilde{C} / \tilde{M}$ and so that the morphism $g: C \rightarrow X$ extends to $\tilde{g}: \tilde{C} \rightarrow X$.

For the family $\tilde{g}: \tilde{C} \rightarrow X$, we form the similarly defined homomorphism

$$
\tilde{\Theta}: \omega_{\tilde{C} / \tilde{M}}^{\vee} \longrightarrow \Omega_{\tilde{C} / \tilde{M}}
$$

Like $\Theta, \tilde{\Theta}$ vanishes away from the singularities of the fibers of $\tilde{C} / \tilde{M}$. On the other hand, since $\tilde{M}$ is smooth and the node $\bar{q} \in \tilde{C}_{\tilde{\xi}}$ is smoothed in the family $\tilde{C} / \tilde{M}$, $\Omega_{\tilde{C} / \tilde{M}}$ has no torsion near $\bar{q}$. Therefore, the fact that $\tilde{\Theta}$ vanishes away from the singular points of the fibers of $\tilde{C} / \tilde{M}$ implies that $\tilde{\Theta}$ vanishes near $\tilde{q}$.

Finally, since $\left.\Theta\right|_{C_{\bar{\xi}}}=\left.\tilde{\Theta}\right|_{C_{\bar{\xi}}}$, we conclude that $\Theta$ vanishes at $q \in \mathcal{C}$. Since $q \in \mathcal{C}$ is an arbitrary node, this shows that $\Theta=0$. This proves that the composite in the statement of the lemma vanishes.

Corollary 6.2. The homomorphism $\sigma_{\theta}^{\text {rel }}$ lifts to a homomorphism $\sigma_{\theta}: \mathcal{O} b_{\mathcal{M}} \rightarrow$ $\mathcal{O}_{\mathcal{M}}$.

Proof. This follows from Lemma 6.1 and the exact sequence 6.4

Definition 6.3. A stable map $u: C \rightarrow X$ is called $\theta$-null if the composite

$$
u^{*}(\hat{\theta}) \circ d u:\left.\left.T_{C_{\mathrm{reg}}} \longrightarrow u^{*} T_{X}\right|_{C_{\mathrm{reg}}} \longrightarrow u^{*} \Omega_{X}\right|_{C_{\mathrm{reg}}}
$$

is trivial over the regular locus $C_{\text {reg }}$ of $C$.

Proposition 6.4. Any holomorphic two-form $\theta \in H^{0}\left(\Omega_{X}^{2}\right)$ on a smooth quasiprojective variety $X$ induces a homomorphism $\sigma_{\theta}: \mathcal{O b}_{\mathcal{M}} \longrightarrow \mathcal{O}_{\mathcal{M}}$ of the obstruction sheaf $\mathcal{O} b_{\mathcal{M}}$ of the moduli of stable morphisms $\mathcal{M}=\overline{\mathcal{M}}_{g, n}(X, \beta)$. The homomorphism $\sigma$ is surjective away from the set of $\theta$-null stable maps in $\mathcal{M}$.

Proof. Only the last part needs to be proved. Since $\sigma_{\theta}$ is the lift of $\sigma_{\theta}^{\text {rel }}$, it is surjective at $[u: C \rightarrow X] \in \mathcal{M}$ if and only if $\sigma_{\theta}^{\text {rel }}$ is surjective at $[u]$. Since the latter at $[u]$ is the composition

$$
H^{1}\left(C, u^{*} T_{X}\right) \stackrel{u^{*} \hat{\theta}}{\longrightarrow} H^{1}\left(C, u^{*} \Omega_{X}\right) \longrightarrow H^{1}\left(C, \Omega_{C}\right) \longrightarrow H^{1}\left(C, \omega_{C}\right)=\mathbb{C},
$$

its Serre dual is

$$
\mathbb{C}=H^{0}\left(C, \mathcal{O}_{C}\right) \longrightarrow H^{0}\left(f^{*} T_{X} \otimes \omega_{C}\right) \longrightarrow H^{0}\left(f^{*} \Omega_{X} \otimes \omega_{C}\right) .
$$


Because $\mathcal{O}_{C}$ is generated by global sections, the composite of the above sequence is trivial if and only if the composite

$$
\left.\left.\left.T_{C} \otimes \omega_{C}\right|_{C_{\mathrm{reg}}} \longrightarrow f^{*} T_{X} \otimes \omega_{C}\right|_{C_{\mathrm{reg}}} \longrightarrow f^{*} \Omega_{X} \otimes \omega_{C}\right|_{C_{\mathrm{reg}}}
$$

is trivial. But this is equivalent to $u$ being $\theta$-null. This proves the proposition.

Using the cosection $\sigma_{\theta}$, we can localize the virtual cycle of $\mathcal{M}$. Let $\mathcal{M}\left(\sigma_{\theta}\right) \subset \mathcal{M}$ be the collection of $\theta$-null stable maps. Clearly, $\mathcal{M}\left(\sigma_{\theta}\right) \subset \mathcal{M}$ is closed. Because $\sigma_{\theta}: \mathcal{O} b_{\mathcal{M}} \rightarrow \mathcal{O}_{\mathcal{M}}$ is surjective away from $\mathcal{M}\left(\sigma_{\theta}\right)$, applying Theorem [5.1, we obtain the localized virtual cycle

$$
[\mathcal{M}]_{\mathrm{loc}}^{\mathrm{vir}} \in A_{*} \mathcal{M}\left(\sigma_{\theta}\right) .
$$

In case $\mathcal{M}\left(\sigma_{\theta}\right)$ is proper, we define the localized GW-invariants as follows. We let ev $: \mathcal{M} \rightarrow X^{n}$ be the evaluation morphism, let $\gamma_{1}, \ldots, \gamma_{n} \in H^{*}(X)$, let $\alpha_{1}, \ldots, \alpha_{n} \in$ $\mathbb{Z}^{\geq 0}$, and let $\psi_{i}$ be the first Chern class of the relative cotangent line bundle of the domain curves at the $i$-th marked point. The localized GW-invariant of $X$ with descendants is defined to be

$$
\left\langle\tau_{\alpha_{1}}\left(\gamma_{1}\right) \cdots \tau_{\alpha_{n}}\left(\gamma_{n}\right)\right\rangle_{g, \beta}^{X, \mathrm{loc}}=\int_{[\mathcal{M}]_{\mathrm{loc}}^{\mathrm{vir}}} \operatorname{ev}^{*}\left(\gamma_{1} \times \cdots \times \gamma_{n}\right) \cdot \psi_{1}^{\alpha_{1}} \cdots \psi_{n}^{\alpha_{n}} .
$$

In case $X$ is proper, $\mathcal{M}\left(\sigma_{\theta}\right)$ is automatically proper.

Lemma 6.5. If $X$ is proper, the localized $G W$-invariant coincides with the ordinary $G W$-invariant of $X$.

Proof. This follows from the last statement in Theorem 5.1

A corollary of this is the following generalization of the vanishing results of J. Lee and T. Parker [20, 21] for compact algebraic surfaces.

Corollary 6.6 (First vanishing). Let $X$ be a smooth projective variety endowed with a holomorphic two-form $\theta$. The virtual cycle of the moduli of stable morphisms $\overline{\mathcal{M}}_{g, n}(X, \beta)$ is trivial unless the class $\beta$ can be represented by a $\theta$-null stable morphism.

Proof. Let $\mathcal{M}=\overline{\mathcal{M}}_{g, n}(X, \beta)$. Suppose $\beta$ cannot be represented by a $\theta$-null stable morphism, i.e., $\mathcal{M}\left(\sigma_{\theta}\right)=\emptyset$. Then obviously $[\mathcal{M}]_{\text {loc }}^{\text {vir }}=0$. Since $[\mathcal{M}]^{\text {vir }}$ is the image of $[\mathcal{M}]_{\mathrm{loc}}^{\mathrm{vir}}$ in $A_{*} \mathcal{M},[\mathcal{M}]^{\mathrm{vir}}=0$.

A generalization of the second vanishing of Lee-Parker is as follows. Since $\hat{\theta}$ is a homomorphism from $T_{X} \rightarrow \Omega_{X}$, $\operatorname{det} \hat{\theta}$ can be viewed as a section of the line bundle $K_{X}^{\otimes 2}$. Let $D=(\operatorname{det} \hat{\theta}=0)$. Every $[u, C] \in \mathcal{M}\left(\sigma_{\theta}\right)$ has $u(C) \subset D$. Then an easy argument shows that

Corollary 6.7 (Second vanishing). Let $(X, \theta)$ be as in Corollary 6.6. For $\beta \neq 0$ and $\gamma_{i} \in H^{*}(X)$, the $G W$-invariant $\left\langle\prod \tau_{\alpha_{i}}\left(\gamma_{i}\right)\right\rangle_{g, \beta}^{X}$ vanishes if one of the classes $\gamma_{i}$ is Poincaré dual to a cycle disjoint from $D$.

Further applications of this cycle localization technique will appear in the sequel of this paper. 


\section{Other Applications}

We conclude our paper with comments on other possible applications; some have been worked out and some are under development.

One application is the study of extremal GW-invariants of the Hilbert schemes of points of surfaces. After Beauville, we know that every holomorphic two-form $\theta$ of an algebraic surface $X$ induces a holomorphic two-form on the Hilbert scheme $X^{[k]}$ of $k$-points of $X$. Thus the second vanishing applied to this case gives us vanishing of a lot of the GW-invariants of $X^{[k]}$. For general $X$, we can pick a meromorphic two-form $\theta$. If $\beta \in H_{2}\left(X^{[k]}, \mathbb{Z}\right)$ is an extremal class (i.e., a class in the kernel of $\left.H_{2}\left(X^{[k]}, \mathbb{Z}\right) \rightarrow H_{2}\left(X^{(k)}, \mathbb{Z}\right)\right)$, then the meromorphic $\theta$ induces a meromorphic twoform on $\overline{\mathcal{M}}_{g, n}\left(X^{[k]}, \beta\right)$. This form can be used to study the GW-invariants of $X^{[k]}$. This has been exploited by W-P. Li and the second author of this paper in 23 .

Another application is in the study of Donaldson-Thomas invariants of a CalabiYau threefold. In [15, the authors showed that the modified Kirwan blow-up of the moduli of semistable sheaves has an obstruction theory whose obstruction sheaf has a regular surjective cosection. Using this cosection, we can localize the virtual cycle and prove a wall-crossing formula using the master space construction and $\mathbb{C}^{*}$-localization. In comparison with Joyce's proof of the wall-crossing formula, our proof does not use the Chern-Simons functional and thus can possibly apply to wider classes of moduli of derived objects over Calabi-Yau three-folds.

Another potential application is the study of the GW-invariants of a three-fold $X$ that is a $\mathbb{P}^{1}$-bundle over a surface $S$ equipped with a holomorphic two-form $\theta$. The two-form $\theta$ on $S$ pulls back to a two-form of $X$. Since the geometry of this two-form is explicit, one hopes that one can essentially reduce the study of the $\mathrm{GW}$-invariants of $X$ to a ruled surface over the canonical divisor $D \subset S$. It will be interesting to see some part of this carried out in the near future.

Addendum. The cosection localized virtual cycle has been applied to construct the GW-invariants of stable morphisms with fields [6]. This is the all genus generalization of the $g=0$ theory in super-string theories. The cosection localized virtual cycle can also give an algebro-geometric approach to FJRW-invariants (Fan-JarvisRuan-Witten) of spin curves with fields.

In 13, 14, the authors derive a degeneration formula for certain localized GromovWitten invariants and use it to prove a conjecture of Maulik-Pandharipande [27. on degree two Gromov-Witten invariants of surfaces.

In [5], the first author of this paper and H.-L. Chang use cosection localization to prove a conjecture of Dürr, Kabanov, and Okonek [9], which gives an algebraic geometric definition of Seiberg-Witten invariants of any smooth projective surface.

\section{Appendix A. Analytic analogue of localized Gysin maps}

In the case of schemes over $\mathbb{C}$, we can construct an analytic analogue of the localized Gysin map by picking a smooth $\left(C^{\infty}\right)$ section of a bundle and intersecting with the cycle representative. This was the original approach adopted in [12]. Due to its potential applications, we include it here. We will also prove the equivalence of the two constructions.

For simplicity, we will work out the case of schemes. The case of orbifolds (DM stacks) is similar, using multiple sections. Let $\pi: E \rightarrow M$ be a rank $r$ holomorphic vector bundle over a reduced scheme $M$, let $U \subset M$ be an open subset, and let 
$\sigma:\left.E\right|_{U} \rightarrow \mathcal{O}_{U}$ be a surjective homomorphism. We denote $M(\sigma)=M \backslash U$ and we denote by $E(\sigma)$ the kernel cone of $\sigma$, as defined earlier. We assume $M(\sigma)$ is compact.

We first pick a splitting of $\sigma$ over $U$. Because $\sigma$ is surjective, possibly by picking a hermitian metric on $E$ we can find a smooth section $\check{\sigma} \in C^{\infty}\left(\left.E\right|_{U}\right)$ so that $\sigma \circ \check{\sigma}=1$. Next, we pick an analytic neighborhood $V$ of $M(\sigma) \subset M$ such that $V$ has compact (analytic) closure in $M$ and the homomorphism

$$
\imath_{*}: H_{*}(M(\sigma), \mathbb{Z}) \stackrel{\cong}{\longrightarrow} H_{*}(V, \mathbb{Z})
$$

induced by the inclusion $\imath: M(\sigma) \rightarrow V$ is an isomorphism. Because $M$ is algebraic and $M(\sigma)$ is compact, such a neighborhood $V$ always exists $([26])$. We then extend $\left.\check{\sigma}\right|_{M-V}$ to a smooth section $\check{\sigma}_{\text {ex }} \in C^{\infty}(E)$ and pick a smooth function $\rho: M \rightarrow \mathbb{R}^{>0}$ so that $\xi=\rho \cdot \check{\sigma}_{e x} \in C^{\infty}(E)$ is a small perturbation of the zero section of $E$.

Now let $B \subset E(\sigma)$ be a complex $d$-dimensional closed subvariety. By fixing a stratification of $B$ and of $M$ by complex subvarieties, we can choose the extension $\check{\sigma}_{e x}$ and the function $\rho$ so that the section $\xi$ intersects $B$ transversely. As a consequence, the intersection $B \cap \xi$, which is of pure dimension, has no real codimension 1 strata. Hence, it defines a closed oriented Borel-Moore cycle in $E$.

But on the other hand, since $\left.\sigma \circ \xi\right|_{M-V}=\rho \in C^{\infty}(M-V), \xi$ is disjoint from $B$ over $M-V$. Thus $\left.B \cap \xi \subset E\right|_{V}$. Adding the fact that $V$ has compact (analytic) closure in $M, \pi(B \cap \xi)$ defines a closed chain in $V$ and thus defines a homology class $[\pi(B \cap \xi)] \in H_{2 d-2 r}(V, \mathbb{Z})$. Finally, applying the inverse of $\iota_{*}$, we define

$$
s_{E, \sigma}^{!, \text {an }}([B])=\imath_{*}^{-1}([\pi(B \cap \xi)]) \in H_{2 d-2 r}(M(\sigma), \mathbb{Z}) .
$$

Applying the standard transversality argument, one easily shows that this class is independent of the choice of $V$ and the section $\xi$; thus it only depends on the cycle $B$ we begin with.

Definition-Proposition A.1. The map $s_{E, \sigma}^{!, \text {an }}: Z_{d} E(\sigma) \rightarrow H_{2 d-2 r}(M(\sigma), \mathbb{Z})$ so defined descends to a homomorphism

$$
s_{E, \sigma}^{!, \text {an }}: A_{d} E(\sigma) \longrightarrow H_{2 d-2 r}(M(\sigma), \mathbb{Z}),
$$

which we call the analytic localized Gysin map. Furthermore, via the cycle-tohomology homomorphism $c l: A_{k} M(\sigma) \rightarrow H_{2 k}(M(\sigma), \mathbb{Z})$, the two versions of localized Gysin maps coincide, i.e., $c l \circ s_{E, \sigma}^{!}=s_{E, \sigma}^{!, \text {an }}$.

Proof. The proof that $s_{E, \sigma}^{!, \text {an }}$ preserves rational equivalence is standard and will be omitted. We now prove the comparison by using the notation of Section 2. If $\left.B \subset E\right|_{M(\sigma)}$, the result follows directly from [10, Lemma $19.2(\mathrm{~d})$ ]. So we suppose $B \cap G \neq \emptyset$. We choose a $\sigma$-regularizing morphism $\rho: X \rightarrow M$ and a closed integral $\tilde{B} \subset \tilde{G}$ such that $\tilde{\rho}_{*}[\tilde{B}]=k \cdot[B]$ where $\tilde{G}$ is the kernel of $\tilde{\sigma}: \tilde{E} \rightarrow \mathcal{O}_{X}(D)$ with $\tilde{E}=\rho^{*} E$ and $\tilde{\rho}: \tilde{E} \rightarrow E$ is induced from $\rho$.

Observe that the intersections $\xi \cap B$ for defining $s_{E, \sigma}^{!, \text {an }}[B]$ and $D \cdot s_{\tilde{G}}^{!}[\tilde{B}]$ for defining $s_{E, \sigma}^{!}[B]$ take place in the tubular neighborhood $V$ of $M(\sigma)$ in the sense that if we replace $M$ by $V$ and $X$ by the inverse image of $V$ and so on, we get the same classes. So for the purpose of the comparison result, we may assume that $M=V$ is a tubular neighborhood of $M(\sigma)$. 
Consider the fiber square of zero sections

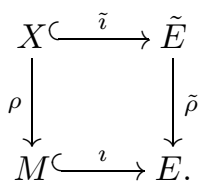

By [10, Theorem 6.2],

$$
\rho_{*}\left(s_{\tilde{E}}^{!}[\tilde{B}]\right)=s_{E}^{!} \tilde{\rho}_{*}[\tilde{B}]=k \cdot s_{E}^{!}[B] .
$$

By the excess intersection formula [10, Theorem 6.3],

$$
\rho_{*}\left(D \cdot s_{\tilde{G}}^{!}[\tilde{B}]\right)=\rho_{*}\left(s_{\tilde{E}}^{!}[\tilde{B}]\right)=k \cdot s_{E}^{!}[B] .
$$

Applying the cycle-to-homology homomorphism $c l$, we obtain

$$
\operatorname{cl}\left(s_{E, \sigma}^{!}[B]\right)=k^{-1} \cdot \operatorname{cl}\left(\rho_{*}\left(D \cdot s_{\tilde{G}}^{!}[\tilde{B}]\right)\right)=\operatorname{cl}\left(s_{E}^{!}[B]\right) .
$$

By [10, Lemma $19.2(\mathrm{~d})]$ again, this equals $u_{\imath} \cap \operatorname{cl}([B])$, where $u_{\imath} \in H^{2 r}(E, E-M)$ is the orientation class of $E$. By standard arguments, the cap product of the orientation class is the same as intersecting $B$ with a transversal perturbation $\xi$ of the zero section. This proves Definition-Proposition A.1.

\section{ACKNOWLEDGMENTS}

The first author is grateful to the Stanford mathematics department for support and hospitality while he was visiting during the academic year 2005/2006. The authors thank J. Lee and T. Parker for stimulating questions and for pointing out several oversights in the authors' previous draft. The authors are also grateful to the referee(s) for careful reading and helpful comments.

\section{REFERENCES}

[1] Kai Behrend, Donaldson-Thomas type invariants via microlocal geometry, Ann. of Math. (2) 170 (2009), no. 3, 1307-1338, DOI 10.4007/annals.2009.170.1307. MR2600874|(2011d:14098)

[2] K. Behrend and B. Fantechi, The intrinsic normal cone, Invent. Math. 128 (1997), no. 1, 45-88, DOI 10.1007/s002220050136. MR1437495(98e:14022)

[3] Jim Bryan and Naichung Conan Leung, The enumerative geometry of K3 surfaces and modular forms, J. Amer. Math. Soc. 13 (2000), no. 2, 371-410 (electronic), DOI 10.1090/S08940347-00-00326-X. MR.1750955 (2001i:14071)

[4] Jim Bryan and Naichung Conan Leung, Generating functions for the number of curves on abelian surfaces, Duke Math. J. 99 (1999), no. 2, 311-328, DOI 10.1215/S0012-7094-99-099118. MR1708022 (2000m:14052)

[5] Huai-Liang Chang and Young-Hoon Kiem Poincaré invariants are Seiberg-Witten invariants. To appear in Geometry and Topology, arXiv:1205.0848.

[6] Huai-Liang Chang and Jun Li, Gromov-Witten invariants of stable maps with fields, Int. Math. Res. Not. IMRN 18 (2012), 4163-4217. MR2975379

[7] Huai-Liang Chang and Jun Li. In preparation.

[8] S. K. Donaldson, Yang-Mills invariants of four-manifolds, Geometry of low-dimensional manifolds, 1 (Durham, 1989), London Math. Soc. Lecture Note Ser., vol. 150, Cambridge Univ. Press, Cambridge, 1990, pp. 5-40. MR1171888 (93f:57040)

[9] Markus Dürr, Alexandre Kabanov, and Christian Okonek, Poincaré invariants, Topology 46 (2007), no. 3, 225-294, DOI 10.1016/j.top.2007.02.004. MR2319736 (2008b:14006)

[10] William Fulton, Intersection theory, 2nd ed., Ergebnisse der Mathematik und ihrer Grenzgebiete. 3. Folge. A Series of Modern Surveys in Mathematics [Results in Mathematics and Related Areas. 3rd Series. A Series of Modern Surveys in Mathematics], vol. 2, SpringerVerlag, Berlin, 1998. MR1644323 (99d:14003) 
[11] C. Faber and R. Pandharipande, Hodge integrals and Gromov-Witten theory, Invent. Math. 139 (2000), no. 1, 173-199, DOI 10.1007/s002229900028. MR1728879 (2000m:14057)

[12] Young-Hoon Kiem and Jun Li. Gromov-Witten invariants of varieties with holomorphic 2forms. ArXiv:0707.2986.

[13] Young-Hoon Kiem and Jun Li, Low degree GW invariants of spin surfaces, Pure Appl. Math. Q. 7 (2011), no. 4, Special Issue: In memory of Eckart Viehweg, 1449-1475. MR2918169

[14] Young-Hoon Kiem and Jun Li, Low degree GW invariants of surfaces II, Sci. China Math. 54 (2011), no. 8, 1679-1706, DOI 10.1007/s11425-011-4258-x. MR2824966 (2012h:14135)

[15] Young-Hoon Kiem and Jun Li, A wall crossing formula of Donaldson-Thomas invariants without Chern-Simons functional, Asian J. Math. 17 (2013), no. 1, 63-94.

[16] Andrew Kresch, Cycle groups for Artin stacks, Invent. Math. 138 (1999), no. 3, 495-536, DOI 10.1007/s002220050351. MR1719823(2001a:14003)

[17] Bumsig Kim, Andrew Kresch, and Tony Pantev, Functoriality in intersection theory and a conjecture of Cox, Katz, and Lee, J. Pure Appl. Algebra 179 (2003), no. 1-2, 127-136, DOI 10.1016/S0022-4049(02)00293-1. MR1958379 (2003m:14088)

[18] Gérard Laumon and Laurent Moret-Bailly, Champs algébriques, Ergebnisse der Mathematik und ihrer Grenzgebiete. 3. Folge. A Series of Modern Surveys in Mathematics [Results in Mathematics and Related Areas. 3rd Series. A Series of Modern Surveys in Mathematics], vol. 39, Springer-Verlag, Berlin, 2000 (French). MR.1771927 (2001f:14006)

[19] Junho Lee, Family Gromov-Witten invariants for Kähler surfaces, Duke Math. J. 123 (2004), no. 1, 209-233, DOI 10.1215/S0012-7094-04-12317-6. MR.2060027 (2005d:53141)

[20] Junho Lee, Holomorphic 2-forms and vanishing theorems for Gromov-Witten invariants, Canad. Math. Bull. 52 (2009), no. 1, 87-94, DOI 10.4153/CMB-2009-011-1. MR2494314 (2010j:53188)

[21] Junho Lee and Thomas H. Parker, A structure theorem for the Gromov-Witten invariants of Kähler surfaces, J. Differential Geom. 77 (2007), no. 3, 483-513. MR2362322(2010b:53159)

[22] Jun Li, A note on enumerating rational curves in a K3 surface, Geometry and nonlinear partial differential equations (Hangzhou, 2001), AMS/IP Stud. Adv. Math., vol. 29, Amer. Math. Soc., Providence, RI, 2002, pp. 53-62. MR.1926435 (2003g:14072)

[23] Jun Li and Wei-Ping Li, Two point extremal Gromov-Witten invariants of Hilbert schemes of points on surfaces, Math. Ann. 349 (2011), no. 4, 839-869, DOI 10.1007/s00208-010-0542-2. MR2777035 (2012d:14098)

[24] Jun Li and Gang Tian, Virtual moduli cycles and Gromov-Witten invariants of algebraic varieties, J. Amer. Math. Soc. 11 (1998), no. 1, 119-174, DOI 10.1090/S0894-0347-98-002501. MR:1467172(99d:14011)

[25] Jun Li and Gang Tian, Comparison of algebraic and symplectic Gromov-Witten invariants, Asian J. Math. 3 (1999), no. 3, 689-728. MR1793677 (2001i:53155)

[26] John Mather, Notes on topological stability, Bull. Amer. Math. Soc. (N.S.) 49 (2012), no. 4, 475-506, DOI 10.1090/S0273-0979-2012-01383-6. MR2958928

[27] D. Maulik and R. Pandharipande, New calculations in Gromov-Witten theory, Pure Appl. Math. Q. 4 (2008), no. 2, Special Issue: In honor of Fedor Bogomolov., 469-500. MR 2400883 (2009d:14073)

[28] D. Maulik, R. Pandharipande, and R. P. Thomas, Curves on K3 surfaces and modular forms, J. Topol. 3 (2010), no. 4, 937-996, DOI 10.1112/jtopol/jtq030. With an appendix by A. Pixton. MR2746343(2012b:14111)

[29] A. Okounkov and R. Pandharipande, Quantum cohomology of the Hilbert scheme of points in the plane, Invent. Math. 179 (2010), no. 3, 523-557, DOI 10.1007/s00222-009-0223-5. MR2587340(2011b:14124)

[30] Angelo Vistoli, Intersection theory on algebraic stacks and on their moduli spaces, Invent. Math. 97 (1989), no. 3, 613-670, DOI 10.1007/BF01388892. MR1005008 (90k:14004)

Department of Mathematics and Research Institute of Mathematics, Seoul National

University, SEOUl 151-747, Korea

E-mail address: kiem@math.snu.ac.kr

Department of Mathematics, Stanford University, Stanford, California 94305

E-mail address: jli@math.stanford.edu 